\title{
Emergence of Resistance to Fungicides: The Role of Fungicide Dose
}

\author{
Alexey Mikaberidze, Neil Paveley, Sebastian Bonhoeffer, and Frank van den Bosch
}

First author: Plant Pathology Group, Institute of Integrative Biology, ETH Zurich, LFW, Zurich, CH-8092, Switzerland; second author: ADAS, Duggleby YO17 8BP, United Kingdom; third author: Theoretical Biology, Institute of Integrative Biology, ETH Zurich, CHN, Zurich, CH-8092; and fourth author: Rothamsted Research, Harpenden, AL5 2JQ, United Kingdom.

Accepted for publication 16 December 2016.

\begin{abstract}
Resistance to antimicrobial drugs allows pathogens to survive drug treatment. The time taken for a new resistant mutant to reach a population size that is unlikely to die out by chance is called "emergence time." Prolonging emergence time would delay loss of control. We investigate the effect of fungicide dose on the emergence time in fungal plant pathogens. A population dynamical model is combined with doseresponse data for Zymoseptoria tritici, an important wheat pathogen. Fungicides suppress sensitive pathogen population. This has two effects. First, the rate of appearance of resistant mutants is reduced, hence the emergence takes longer. Second, more healthy host tissue becomes available for resistant mutants, increasing their chances to invade and accelerates emergence. In theory, the two competing effects may lead to a

non-monotonic dependence of the emergence time on fungicide dose that exhibits a minimum. But according to field data, fungicides are unable to reduce the fungicide-sensitive population strongly enough even at high doses. Hence, for full resistance over realistic ranges of pathogen's life history and fungicide dose-response parameters, emergence time decreases monotonically with increasing dose. For partial resistance, there can be cases within a limited parameter range, when emergence decelerates at higher doses.

Additional keywords: disease control, epidemiology, fungicide resistance, host-pathogen interaction, infectious disease, mathematical model, plant pathogens.
\end{abstract}

Fungicide resistance develops in two phases: the emergence phase and the selection phase (Milgroom 1990; van den Bosch and Gilligan 2008; van den Bosch et al. 2011; zur Wiesch et al. 2011). First, resistant mutants arise through mutation and invade the pathogen population (emergence phase). During this phase, the size of the fungicide-resistant pathogen subpopulation is small, so that it can go extinct due to stochastic fluctuations. The emergence phase starts when the new fungicide is introduced and lasts until the resistant mutants build up a substantial subpopulation that is unlikely to die out by chance. (We describe the typical duration of this phase as the emergence time.) After successful emergence, the frequency of resistance increases due to selection imposed by a fungicide in a deterministic fashion (selection phase).

Fungicide producers generally recommend to apply high doses that correspond to the label recommended dose as a strategy to manage fungicide resistance (Brent and Hollomon 2007). (It is illegal to apply doses higher than the label recommended dose.) Yet, we expect higher fungicide doses to induce stronger selection for resistance and accelerate the increase in the frequency of resistant strains. Indeed, a number of experimental and theoretical studies have clearly demonstrated that higher doses lead to faster development of fungicide resistance in the selection phase in vast majority of cases (van den Bosch et al. 2014).

But the emergence of fungicide resistance can be either accelerated or delayed by increasing the fungicide dose. An increase in fungicide dose suppresses the fungicide-sensitive pathogen population, which leads to two competing effects. First, this suppression decreases the number of resistance mutations appearing in the

Corresponding author: A. Mikaberidze;

E-mail address: alexey.mikaberidze@env.ethz.ch

Third and fourth authors share the last author position. population per unit time, which delays emergence. Second, the suppression of the sensitive strain makes it easier for resistant mutants to survive, because of reduced competition for the healthy host tissue between the two strains. This second effect accelerates the emergence of resistance. Theoretically, the interplay of these two effects may lead to a non-monotonic dependence of the time until resistance emergence on the fungicide dose (Day and Read 2016; Kouyos et al. 2014). Which of these two effects plays a dominant role under realistic conditions in plant pathogen populations is still an open question. To our knowledge, no experimental studies (neither laboratory nor field experiments) have been published that investigate the effect of dose on the emergence time. Hobbelen et al. (2014) studied the effect of fungicide dose on the emergence time using a stochastic mathematical model. The model (Hobbelen et al. 2014) was parameterized for the case of pyraclostrobin (strobilurin fungicide class) controlling Zymoseptoria tritici (formerly Mycosphaerella graminicola). Resistance to fungicides from this class is conferred by a single mutation and gives the pathogen full protection from the fungicide (absolute resistance). The study (Hobbelen et al. 2014) reported for this specific pathogen-fungicide combination that the emergence accelerates at higher fungicide doses.

How general is this outcome? Can there be realistic scenarios when the emergence time increases with the fungicide dose? To address these questions, we devised a mathematical model of stochastic emergence of fungicide resistance that is specific to plant pathogen populations, but simple enough to be analytically tractable. In this way, we determined the outcomes in the full range of epidemiological parameters that characterize the host-pathogen interaction and the dose-response parameters that characterize the effect of the fungicide. As a case study, we also considered emergence of fungicide resistance in Z. tritici, which is the most damaging wheat pathogen in Europe (Jorgensen et al. 2014) and an important pathogen worldwide (Dean et al. 2012). We combined our model with outcomes of field experiments on dose response of this pathogen to several important fungicides to determine parameter ranges over which increasing fungicide dose within the range of legal doses delays or

(C) 2017 The American Phytopathological Society 
accelerates emergence of resistance for these host-pathogen-fungicide combinations.

In contrast to Hobbelen et al. (2014), here we also considered the case of partial resistance, when a resistance mutation gives the pathogen only partial protection from the fungicide and several successive mutations are needed to acquire considerable levels of resistance. This is the case for azoles, fungicides that are widely used to protect different crops from a range of fungal diseases (Cools and Fraaije 2013). Our results demonstrate that for partial resistance (to azoles), there can be cases within a limited parameter range, when emergence decelerates at higher doses.

\section{MATERIALS AND METHODS}

The pathogen population consists of a sensitive and a resistant strain, which we will denote by subscripts $s$ and $r$. The dynamics of the sensitive strain are described by deterministic equations, as we consider the sensitive subpopulation size to be high enough such that stochastic effects are negligible. The dynamics of the resistant strain are described by stochastic processes, because here we are interested in the process of emergence of resistance from single mutant individuals, in which case stochastic effects become important (Caswell 2001; Shaw 1994).

The dynamics of the sensitive strain. We use the following system of ordinary differential equations to describe the dynamics of the sensitive strain

$$
\begin{gathered}
\frac{d H}{d t}=r_{H}\left(K-H-I_{s}\right)-\beta_{s} I_{s} H \\
\frac{d I_{s}}{d t}=\beta_{s} I_{s} H-\mu I_{s}+\delta_{s}
\end{gathered}
$$

where $H$ represents the density of healthy hosts and $I_{s}$ is the density of hosts infected with the sensitive pathogen strain. Here, density means leaf area per unit area of land. The first term on the right side of equation 1 describes the host growth, according to the "monomolecular" equation that is often used in plant pathology (Cunniffe and Gilligan 2010; Madden et al. 2006b). Healthy hosts $H$ initially grow linearly over time with the rate $r_{H} K$. This growth is limited by a "carrying capacity" $K$, which accounts for space or nutrient limitations. Healthy hosts can become infected with transmission rate $\beta_{s}$. This is a compound parameter given by the product of the sporulation rate of the infectious tissue, the probability that a spore lands on the appropriate host tissue and the probability that a spore causes a new infection (infection efficiency). Infectious host tissue loses its infectivity at a rate $\mu$, where $\mu^{-1}$ is the characteristic infectious period. We assume a constant rate of pathogen immigration $\delta_{s}$. Population dynamical model equation 1 and equation 2 is similar to the models described in Hall et al. (2007) and Mikaberidze et al. (2014, 2015).

The dynamics of the resistant strain. Resistant mutants are quantified using $I_{r}$, the number of lesions of the resistant strain. They arise through de novo mutations and this process is described by a stochastic birth-death process with the birth rate $B(t)=\beta_{r} H(t)$ and the death rate $\mu$ (the rate of at which an infectious lesion reaches the end of its infectious period plays the role of the death rate in this context). Here, $\beta_{r}$ is the transmission rate of the resistant pathogen strain.

The model of fungicide action. We take into account the effect of the fungicide on the transmission rate of the sensitive strain using the expression

$$
\beta_{s}=\beta\left[1-\varepsilon_{s}(D)\right]
$$

where the effect of the fungicide on the transmission rate, $\varepsilon_{s}(D)$, is given by the Hill function

$$
\varepsilon_{s}(D)=\varepsilon_{m} \frac{D}{D+D_{50}}
$$

and describes the proportion by which the transmission rate is reduced by the fungicide. In equation $4, D$ is the fungicide dose, $\varepsilon_{m}$ is the maximal fungicide effect, and $D_{50}$ the shape parameter that quantifies the dose at which half of the maximum effect is achieved. The Hill function generally describes empirical dose-response curves well.

The transmission rate of the resistant strain, $\beta_{r}$, can be reduced by the fungicide in the case of partial resistance. Hence,

$$
\beta_{r}=\beta(1-\theta)\left[1-\varepsilon_{r}(D)\right]
$$

where the effect of the fungicide is quantified by

$$
\varepsilon_{r}(D)=\alpha_{1} \varepsilon_{m} \frac{D}{D+D_{50} / \alpha_{2}}
$$

Partial resistance may affect the dose-response dependence in two different ways: (i) by decreasing the maximum fungicide effect, $\varepsilon_{m} \rightarrow$ $\alpha_{1} \varepsilon_{m}$, where $0<\alpha_{1}<1$ (we will term this partial resistance type 1 or PR1) and (ii) by decreasing the slope of the fungicide effect with dose, $D_{50} \rightarrow D_{50} / \alpha_{2}$, where $0<\alpha_{2}<1$ (partial resistance type 2 or PR2). $\theta$ in equation 5 quantifies the fitness cost of resistance. We found that inclusion of the fitness cost does not have any major effect on the conclusions of this study, hence we set $\theta=0$ throughout the main text, but for completeness we also derived most important equations also at $\theta>0$ (in Appendix A and B). When resistance is absolute, $\varepsilon_{r}(D)=0$ and therefore, $\beta_{r}=\beta$.

Emergence of resistance. The rate of resistance emergence consists of two factors. First is the rate of appearance of resistant mutants, $r_{a p p}$. Second is the probability that a single resistant mutant will survive and establish a subpopulation large enough not to go extinct by chance, $P_{\text {surv }}$. Hence, the rate of emergence is given by (Appendix A describes mathematical details)

$$
\rho(D)=r_{\text {app }}(D) P_{\text {surv }}(D)
$$

where

$$
\begin{gathered}
r_{a p p}(D)=m \beta_{s} \frac{o}{\psi} I_{s}^{*} H^{*} \\
P_{\text {surv }}(D)=1-\frac{R_{0 s}}{R_{0 r}}=\frac{\varepsilon_{s}(D)-\varepsilon_{r}(D)}{1-\varepsilon_{r}(D)}
\end{gathered}
$$

In equation $8, m$ is the probability of mutation from the fungicidesensitive pathogen type to the fungicide-resistant pathogen type, $o$ is the land area occupied by host plants, and $\psi$ is the leaf area of a single lesion. The factor $o / \psi$ in equation 8 converts the density of hosts used in the deterministic part of the model, to the number of individuals (counted as lesions) used in the stochastic part. It is reasonable to assume that for fungal plant pathogens a transmission event is required for a resistant individual to appear. Hence, $r_{a p p}(D)$ is proportional to the transmission rate of the sensitive strain $\beta_{s}$, to the density of hosts infected by the sensitive strain $I_{s}^{*}$ and to the density of healthy hosts $H^{*}$. Here, $r_{a p p}(D)$ is proportional to $\beta_{s}$, not to $\beta_{r}$. This holds when the fungicide affects the spore production rate. However, if the fungicide affects the infection efficiency, then $r_{a p p}(D)$ should be proportional to $\beta_{r}$. We obtained all the results also for this case (not shown here). There is a slight quantitative difference in the outcomes, but all qualitative conclusions remain the same.

We assumed that the dynamics of the sensitive pathogen strain governed by equation 1 and equation 2 continues over a long enough time to reach the endemic equilibrium (i.e., a stationary state at which the amounts of healthy and infected hosts remain constant over time), i.e., $H(t)=H^{*}, I_{s}(t)=I_{s}^{*}$. 
In equation $9, R_{0 s}=R_{0}\left(1-\varepsilon_{s}\right)$ is the basic reproductive number of the sensitive strain and $R_{0 r}=R_{0}\left(1-\varepsilon_{r}\right)$ is the basic reproductive number of the resistant strain, where $\varepsilon_{s}$ and $\varepsilon_{r}$ are fungicide effects on transmission rates of the sensitive and resistant pathogen strains, correspondingly (equation 4 and equation 6). Here, $R_{0}=\beta K / \mu$ is the pathogen's basic reproductive number in the absence of the fungicide. Basic reproductive number characterizes the reproductive fitness of the pathogen and its value determines whether the epidemic will eventually spread $\left(R_{0}>1\right)$ or die out $\left(R_{0}<1\right)$.

We consider the emergence of resistant mutants to be a homogeneous Poisson process with the rate $\rho(D)$ given by equation 7 . The characteristic time until the emergence of fungicide resistance, $T_{\text {emerg }}$, is then given by the inverse of $\rho(D)$. $T_{\text {emerg }}$ represents the mean waiting time until the first event in the Poisson process.

Using equation 8 and equation 9 and the expressions for the host densities $H^{*}$ and $I_{s}^{*}$, we find

$$
T_{\text {emerg }}=1 / \rho=C \frac{\left[1-\varepsilon_{s}(D)\right]}{\left(R_{0}\left[1-\varepsilon_{s}(D)\right]-1\right)\left[\varepsilon_{s}(D)-\varepsilon_{r}(D)\right]}
$$

where $R_{0}=\beta K / \mu$ is the basic reproductive number of the pathogen in the absence of fungicides and $C=\beta \psi\left(\mu+r_{H}\right) /\left(\right.$ mor $\left._{H} \mu^{2}\right)$. The qualitative behavior of $T_{\text {emerg }}(D)$ does not depend on $C$, since it is merely a proportionality constant, but it does depend on $R_{0}$, the fungicide dose-response parameters $\varepsilon_{m}$ and $D_{50}$, and the fungicide resistance parameters $\alpha_{1}$ and $\alpha_{2}$.

Variables and parameters of the model are listed in Table 1. The values of the parameters used for the $Z$. tritici-wheat pathosystem are provided and justified in Appendix C. In particular, we used empirical data on fungicide dose-response to estimate the fungicide parameters $\varepsilon_{m}$ and $D_{50}$ for five fungicides belonging to three different classes.

\section{RESULTS}

Absolute resistance. We first investigate the dependence of the emergence time, $T_{\text {emerg }}$, on the fungicide dose, $D$, when the resistant pathogen subpopulation is fully protected from the fungicide (i.e., resistance is absolute). Our analysis demonstrates that $T_{\text {emerg }}(D)$ exhibits three qualitatively different regimes (Appendix B). In the first regime $(\mathrm{A})$, the emergence time decreases monotonically with the dose (Fig. 1A). The second (B) and the third (C) regimes share a key feature: $T_{\text {emerg }}(D)$ exhibits a minimum at a dose $D=D_{\text {min }}$ and $T_{\text {emerg }}(D)$ increases with dose above $D_{\text {min }}$, where $D_{\min }$ is given by

$$
D_{\min }=D_{50} \frac{1}{\varepsilon_{m} /\left(1-1 / \sqrt{R_{0}}\right)-1}
$$

But these two regimes differ in the relationship between the dose $D_{\min }$ and the label recommended dose, $D_{\text {rec }}=1$ (which we set to be a unit dose). In regime $\mathrm{B}, D_{\min }>1$, while in regime C, $D_{\min }<1$ (Fig. 1 , compare right panels $\mathrm{B}$ and $\mathrm{C}$ ). This distinction is important from a practical point of view, because it is prohibited to apply fungicide doses higher than the label recommended dose. Thus, regimes $\mathrm{A}$ and B represent the case when $T_{\text {emerg }}(D)$ monotonically decreases in the legal dose range. Hence, within this range increasing dose accelerates resistance emergence. In contrast, in regime $\mathrm{C}$ there is a range of doses within the legal range over which increasing dose delays emergence of resistance. We are interested in conditions under which this regime is realized.

Which of these three regimes manifests in the case of full resistance is determined by shape of the fungicide dose-response curve (equation 4 ), governed by the two dose-response parameters $\varepsilon_{m}$ and $D_{50}$, and the pathogen's basic reproductive number, $R_{0}$. Regime $\mathrm{A}$ is realized at low values of $\varepsilon_{m}$ and high values of $R_{0}$, regime $\mathrm{B}$ is realized at intermediate values of $\varepsilon_{m}$, and regime $\mathrm{C}$ is realized at high values of $\varepsilon_{m}$ and low values of $R_{0}$ (Fig. 1).
Why does $T_{\text {emerg }}(D)$ have a minimum, at high values of $\varepsilon_{m}$ and low values of $R_{0}$ ? The emergence time $T_{\text {emerg }}$ is the inverse of the emergence rate $\rho$ (equation 7), which is the product of the two factors: the rate of mutant appearance $r_{a p p}$ (equation 8) and the probability of building up a population from a single mutant $P_{\text {surv }}$ (equation 9). Here, we consider full resistance $\left(\varepsilon_{r}=0\right)$, where $P_{\text {surv }}(D)$ equals to the effect of the fungicide on the sensitive strain, $\varepsilon_{s}(D)$. Hence, $P_{\text {surv }}(D)$ increases monotonically with dose, $D$, due to increased suppression of sensitive competitors. Consequently, nonmonotonic dependence in $\rho(D)$ and $T_{\text {emerg }}(D)$ is achieved only if $r_{\text {app }}$ exhibits a maximum or decreases with dose. This occurs if the sensitive pathogen subpopulation, $I_{s}^{*}$, is suppressed strongly enough. This is possible at high values of $\varepsilon_{m}$ and low values of $R_{0}$. The threshold value of $\varepsilon_{m}$ above which the minimum in $T_{\text {emerg }}(D)$ occurs is given by

$$
\varepsilon_{m c 1}=1-\frac{1}{\sqrt{R_{0}}}
$$

$\varepsilon_{m c 1}$ increases with $R_{0}$ (Fig. 1, solid curve in left panel), because at higher $R_{0}$ values it is more difficult to suppress the fungicidesensitive pathogen subpopulation with the fungicide. Hence, more resistant mutants appear per unit time as $R_{0}$ increases.

For a dose range to exist where an increasing dose delays resistance, the minimum in $T_{\text {emerg }}(D)$ needs to occur at a dose below the label recommended dose $\left(D_{\min }<1\right)$. To achieve this, $\varepsilon_{m}$ needs to be larger than $\varepsilon_{m c 2}$, where

$$
\varepsilon_{m c 2}=\left(D_{50}+1\right) \frac{\sqrt{R_{0}}-1}{\sqrt{R_{0}}}
$$

While the first threshold, $\varepsilon_{m c 1}$, depends only on $R_{0}$, the second threshold, $\varepsilon_{m c 2}$, depends on both $R_{0}$ and $D_{50}$, because the position, $D_{\text {min }}$, of the minimum in $T_{\text {emerg }}(D)$ is proportional to $D_{50}$ (equation 11).

Thus, our analysis indicates that the minimum in $T_{\text {emerg }}(D)$ can be achieved within the legal range of fungicide doses when the suppression of the sensitive pathogen subpopulation is efficient. This occurs for pathogens with low basic reproductive numbers, $R_{0}$, and for fungicides with low $D_{50}$ and high $\varepsilon_{m}$.

\begin{tabular}{|c|c|c|}
\hline Variables and parameters & Description & Dimension \\
\hline \multicolumn{3}{|l|}{ Variables } \\
\hline$H$ & Healthy host density & $\mathrm{dl}$ \\
\hline$I_{s}$ & $\begin{array}{l}\text { Host density infected by sensitive } \\
\text { pathogens }\end{array}$ & $\mathrm{dl}$ \\
\hline$I_{r}$ & $\begin{array}{l}\text { Number of lesions of the resistant } \\
\text { pathogens }\end{array}$ & dl \\
\hline \multicolumn{3}{|l|}{ Pathosystem parameters } \\
\hline$\beta_{s}$ & $\begin{array}{l}\text { Transmission rate of sensitive } \\
\text { pathogens }\end{array}$ & day $^{-1}$ \\
\hline$\beta_{r}$ & $\begin{array}{l}\text { Transmission rate of resistant } \\
\text { pathogens }\end{array}$ & day $^{-1}$ \\
\hline$\mu^{-1}$ & Average infectious period & days \\
\hline$r_{H}$ & Growth rate of healthy host tissue & day $^{-1}$ \\
\hline$K$ & $\begin{array}{l}\text { Carrying capacity of the healthy } \\
\text { host tissue }\end{array}$ & $\mathrm{dl}$ \\
\hline$R_{0}$ & Basic reproductive number & $\mathrm{dl}$ \\
\hline$\delta_{s}$ & $\begin{array}{l}\text { Immigration rate of sensitive } \\
\text { pathogens }\end{array}$ & day $^{-1}$ \\
\hline$o$ & Land area occupied by host plants & $\mathrm{km}^{2}$ \\
\hline$\psi$ & Leaf area of a single lesion & $\mathrm{km}^{2}$ \\
\hline \multicolumn{3}{|l|}{ Fungicide parameters } \\
\hline$D$ & Fungicide dose & $\mathrm{dl}$ \\
\hline$\varepsilon_{m}$ & Maximum fungicide effect & $\mathrm{dl}$ \\
\hline$D_{50}$ & $\begin{array}{l}\text { Shape parameter of the fungicide } \\
\text { dose-response }\end{array}$ & $\mathrm{dl}$ \\
\hline
\end{tabular}

Pathogen immigration. Influx of fungicide-sensitive pathogen individuals through immigration (modeled as a constant $\delta_{s}$ in

TABLE 1. Important variables and parameters of the model 
equation 2) has two opposing effects. On one hand, it increases the rate of de novo appearance of resistant mutants through mutation, which accelerates emergence of resistance. On the other hand, a larger sensitive subpopulation is more successful in occupying the healthy leaf area. Therefore, sensitives have a competitive advantage compared with resistants. This slows down emergence of resistance. Thus, depending on the parameter regime, immigration can have a different effect on the time until resistance emergence. This is illustrated in Figure 2, from which we see that the effect of immigration on the time until emergence can depend not only on the regime, but also on the fungicide dose (Appendix D provides mathematical details).

In regime A, immigration slows down the emergence of resistance over the whole range of fungicide doses. In this case, the effect of an increased advantage of a larger sensitive subpopulation supersedes the increased rate of appearance of resistant mutants. Here, immigration does not change the qualitative behavior of $T_{\text {emerg }}(D)$ : it decreases monotonically with the dose both with and without immigration.

In contrast, in regimes $\mathrm{B}$ and $\mathrm{C}$, the effect of immigration depends on the fungicide dose. While at low doses immigration slows down the emergence of resistance, at large doses it accelerates emergence. As a result, the minimum in $T_{\text {emerg }}(D)$ in regimes $\mathrm{B}$ and $\mathrm{C}$ becomes less pronounced or even disappears due to immigration (Fig. 2B and $\mathrm{C}$, compare curves).

The key difference between regime $\mathrm{A}$ and regimes $\mathrm{B}$ and $\mathrm{C}$ that determines this behavior is the difference in the value of maximum fungicide effect, $\varepsilon_{m}$. In regime A, $\varepsilon_{m}$ is low enough such that even at large fungicide doses, the sensitive subpopulation is not suppressed enough to decrease the rate of appearance of resistant mutants, $r_{a p p}$. In contrast, in the regimes $\mathrm{B}$ and $\mathrm{C}$, the fungicide efficacy is large enough to suppress the sensitive subpopulation and decrease (regime B) or even bring to zero (regime $\mathrm{C}$ ) the rate of appearance of resistant mutants, $r_{a p p}$.

Thus, immigration can change the qualitative behavior of $T_{\text {emerg }}(D)$ by making the minimum less pronounced or even disappear. This occurs at large $\varepsilon_{m}$ value (regimes B and C) and high doses, when the size of the sensitive population becomes a limiting factor for the emergence of resistance.

Partial resistance. A range of cases is known where resistance to fungicides is only partial, when the resistant pathogen subpopulation is only partially protected from the fungicide. This is the case for example in azoles, an important class of fungicides. Do our conclusions for the case of full resistance also hold for partial resistance? From our analysis we find that this depends on the type of partial resistance: PR1 or PR2 (equation 6 in Materials and Methods; also Appendix E provides mathematical details).

In contrast to full resistance, the fungicide has an effect on the partially resistant pathogen subpopulation. Hence, in the presence of the fungicide, partially resistant mutants are less likely to survive than fully resistant mutants (provided all other factors are equal). As a result, the emergence of partial resistance takes a longer time than emergence of full resistance.

Partial resistance type 1 (PR1). In the case of PR1, only the maximum effect of the fungicide at high doses, $\varepsilon_{m}$, is decreased compared with the sensitive strain (Fig. 3, asymptote shifts in upper left panel). As a result, qualitative features of the dependence $T_{\text {emerg }}(D)$ remain the same as in the case of full resistance (compare Figure 1 and Figure 4). In particular, the dose at which the emergence time, $T_{\text {emerg }}(D)$ is minimal remains unchanged (Fig. 4, right panels).

Partial resistance type 2 (PR2). Interestingly, when partial resistance slows down the increase of the fungicide effect with the dose (PR2, increase in $D_{50}$; Fig. 3, middle left panel), a minimum in $T_{\text {emerg }}(D)$ appears in the whole range of values of $\varepsilon_{m}, D_{50}$ and $R_{0}$. We are interested in the parameter range where the minimum is achieved within a legal dose range, i.e., $D_{\min }<1$. For this reason, we
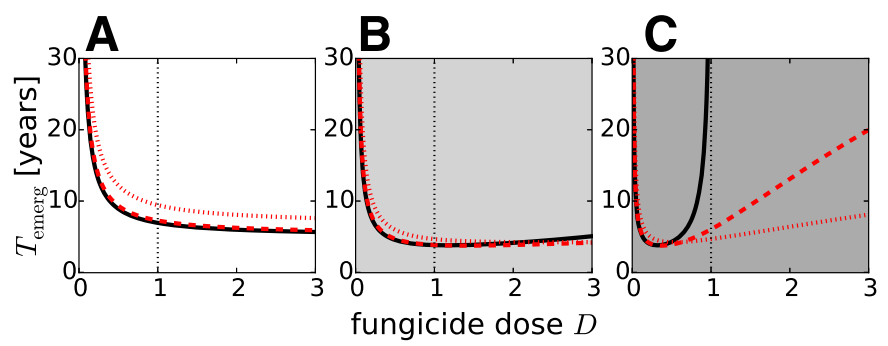

Fig. 2. Effect of immigration on the dose-dependence of the time until emergence of fungicide resistance, $T_{\text {emerg. }}$. The plots show $T_{\text {emerg }}$ versus the fungicide dose, $D$, with immigration (dashed and dotted curves, $\delta_{s}>0$ ) and without immigration (solid curve, $\delta_{s}=0$ ) for the three regimes, $\mathrm{A}, \mathrm{B}$, and $\mathrm{C}$ illustrated in Figure 1. The value of the immigration rate was $\delta_{s}=0.01 \beta I^{*} H^{*}$ (dashed line) and $\delta_{s}=0.1 \beta I^{*} H^{*}$ (dotted line). The curves without immigration (solid line) are the same as in the right panels of Figure 1.
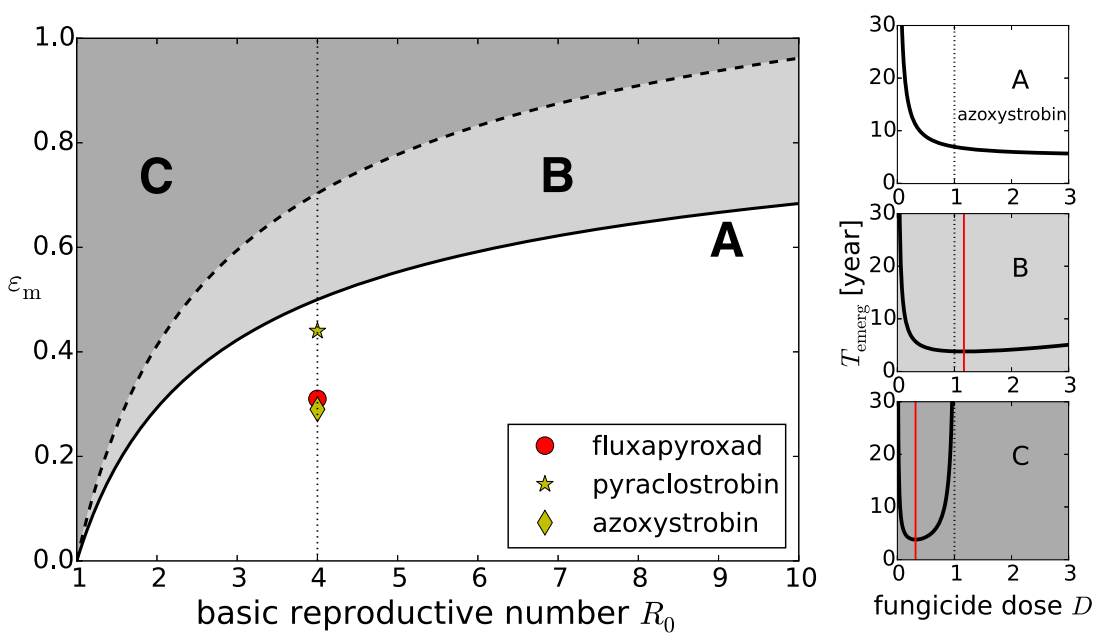

Fig. 1. Mean time until emergence of fungicide resistance, $T_{\text {emerg }}$, can depend on fungicide dose, $D$, in three qualitatively different ways. Regime $\mathrm{A}$ (white): $T_{\text {emerg }}$ decreases monotonically with $D$. Regime B (light gray): $T_{\text {emerg }}$ exhibits a minimum at $D=D_{\min }>1$. Regime C (dark gray): $T_{\text {emerg }}$ exhibits a minimum at $D<1$, where $D$ is measured in units of the label recommended dose, $D_{\text {rec }}$. Left panel: solid curve is plotted using equation 12 , dashed curve is plotted according to equation 13 at $D_{50}=0.4$. Right panels: $T_{\text {emerg }}$ versus $D$ is plotted according to equation 10 at $\varepsilon_{m}=0.29, D_{50}=0.46$ (correspond to azoxystrobin) $(\mathrm{A}) ; \varepsilon_{m}=0.8, D_{50}=$ 0.7 (B); $\varepsilon_{m}=0.97, D_{50}=0.3(\mathrm{C})$. We assumed complete fungicide resistance $\left(\alpha_{1}=0\right)$ and no immigration $\left(\delta_{s}=0\right)$, $C=3.8$. Vertical dotted line indicates the estimate of $R_{0}$ for Zymoseptoria tritici $\left(R_{0}=4.0\right)$ and the points show the estimates of $\varepsilon_{m}$ from field dose-response curves for three fungicides (fluxapyroxad, pyraclostrobin, and azoxystrobin). In right panels, solid red vertical lines indicate the positions of the minimum $D=D_{\text {min }}$ in $T_{\text {emerg }}(D)$; dashed black vertical lines indicate the label recommended dose $D_{\text {rec }}=1$. 
plotted the borders of this range (contours of $D_{\min }=1$ ) versus $\varepsilon_{m}$ and $R_{0}$ at different values of $\alpha_{2}$ in Figure 5. The regions where $D_{\min }<1$ are located to the left from the curves in Figure 5. Figure 6 illustrates the dependence of time until emergence on the fungicide dose for the case of two azole fungicides, tebuconazole and epoxiconazole (next section provides more details).

The range where $D_{\min }<1$ expands with respect to the case of full resistance and includes now also the region of lower values of $\varepsilon_{m}$ (Fig. 5 , compare solid black curve and all other curves in left panel). The dose $D_{\text {min }}$ at which $T_{\text {emerg }}$ has its maximum shifts to lower dosages with increasing fungicide sensitivity $\alpha_{2}$ (Fig. 5, right panels).

Explanation. Partial resistance type 2 differs qualitatively from the cases of full resistance and partial resistance type 1 , because here the minimum in $T_{\text {emerg }}(D)$ occurs in the whole range of parameter values. This is because the probability of mutant survival, $P_{\text {surv }}(D)$, has a maximum in the whole range of parameters due to convergence of the dose-response curves of sensitive and resistant strains at high doses (Fig. 3, middle panels). The probability of mutant survival is proportional to the difference between the fungicide effect on the sensitive strain and the fungicide effect on the resistant strain: $P_{\text {surv }}(D) \propto \varepsilon_{s}(D)-\varepsilon_{r}(D)$ (equation 9). Hence, $P_{\text {surv }}(D)$ first grows from zero with increasing dose, reaches a maximum and declines to zero at large doses (Fig. 3, middle panels).

The minimum in $T_{\text {emerg }}(D)$ that appears in the legal dose range in the case of PR2, is likely to disappear when both types of partial resistance are present together. This is because the shift of the asymptotic value of the fungicide effect quenches the convergence of the dose-response curves that is responsible for the minimum (Fig. 3, lower panels). In summary, (i) partial resistance takes longer to emerge than full resistance (provided that the partially resistant strain and the fully resistant strain are identical in all characteristics, except fungicide resistance). The two types of partial resistance, PR1 and PR2, differ in the way they affect qualitative dependence of the time until emergence on the dose, $T_{\text {emerg }}(D)$. (ii) PR1 does not lead to any qualitative changes in $T_{\text {emerg }}(D)$ compared with full resistance in the whole range of parameters. (iii) When PR2 is present, the dose-response dependencies for the sensitive and the resistant pathogen strains converge at high doses. As a result, the probability of mutant survival exhibits a maximum versus the fungicide dose. Consequently, the parameter range in which $T_{\text {emerg }}(D)$ achieves a minimum at a dose lower than the label recommended dose $\left(D_{\min }<1\right)$ is extended and includes low values of the maximum fungicide effect, $\varepsilon_{m}$. (iv) When both PR1 and PR2 are present simultaneously, the parameter range where $D_{\min }<1$ is diminished because the convergence of the dose-response curves is relaxed.

Case study: Resistance of $\boldsymbol{Z}$. tritici to five fungicides. In two previous sections, we found conditions under which time until resistance emergence, $T_{\text {emerg }}(D)$, exhibits a minimum within the legal range of doses (Fig. 1, regime C). Here, we determine how realistic these condisions are. We relate the results we obtained above to the empirical dose-response data recorded in the field for $Z$. tritici, an important pathogen of wheat and five fungicides belonging to three different classes (according to the mode of action). In particular, we consider two classes of fungicides to which the pathogen may evolve full resistance: strobilurins (pyraclostrobin and azoxystrobin) and succinate dehydrogenase inhibitors (fluxapyroxad), and a class of fungicides known for its wide variety of levels of resistance, the azole fungicides (tebuconazole and epoxiconazole).

First, consider full resistance. The points in Figure 1 indicate estimates of the maximum fungicide effect, $\varepsilon_{m}$, and the basic reproductive number, $R_{0}$, for three fungicides (fluxapyroxad, pyraclostrobin and azoxystrobin). All three points lie below the solid curve (regime A), meaning that the time until emergence decreases monotonically with the dose. Thus, to delay emergence of resistance in these cases, it is better to apply as little fungicide as needed to keep effective control of the pathogen population.

Figure 7 reveals more subtle aspects of this data-model comparison: it shows the same data points and curves, but now in the coordinates of $\varepsilon_{m}$ and $D_{50}\left(R_{0}=4\right.$ is fixed to its estimate for $Z$. tritici $)$. We learn from this figure that in the parameter range, where the estimates for the three fungicides lie (regime A), a minimum in $T_{\text {emerg }}(D)$ cannot be achieved at any values of $D_{50}$. However, if the maximum efficacy, $\varepsilon_{m}$, exceeds a critical value, $\varepsilon_{m c 1}$, (shown using solid horizonal line), low enough values of $D_{50}$ would lead to a minimum in $T_{\text {emerg }}(D)$ within the legal range of doses (regime $\mathrm{C}$ ).

The outcome can be different in the case of azoles and partial resistance. According to our model, the type of partial resistance makes a difference. The dose-response data that we used for azoles characterizes the sensitive strain, as it was collected before the detection of resistance (Paveley et al. 1998). After resistance started to emerge, many strains each with their own level of resistance were likely to increase in frequency. For this reason, the dose-response measurements after detection of resistance do not allow to estimate the fungicide sensitivities $\alpha_{1}$ and $\alpha_{2}$ for individual resistant strains. Therefore, we discuss emergence of azole resistance under different scenarios covering the whole range of possible values of fungicide sensitivities, i.e., $0<\alpha_{1}, \alpha_{2}<1$.

In the case of PR1, the parameter range in which $T_{\text {emerg }}(D)$ has a minimum below the label recommended dose is the same as in the case of full resistance (Fig. 4, above the solid line in left panel). The triangles in Figure 4 indicate estimates of the dose-response parameters for epoxiconazole and tebuconazole. Both of them lie below the solid line. Hence, the time until emergence decreases
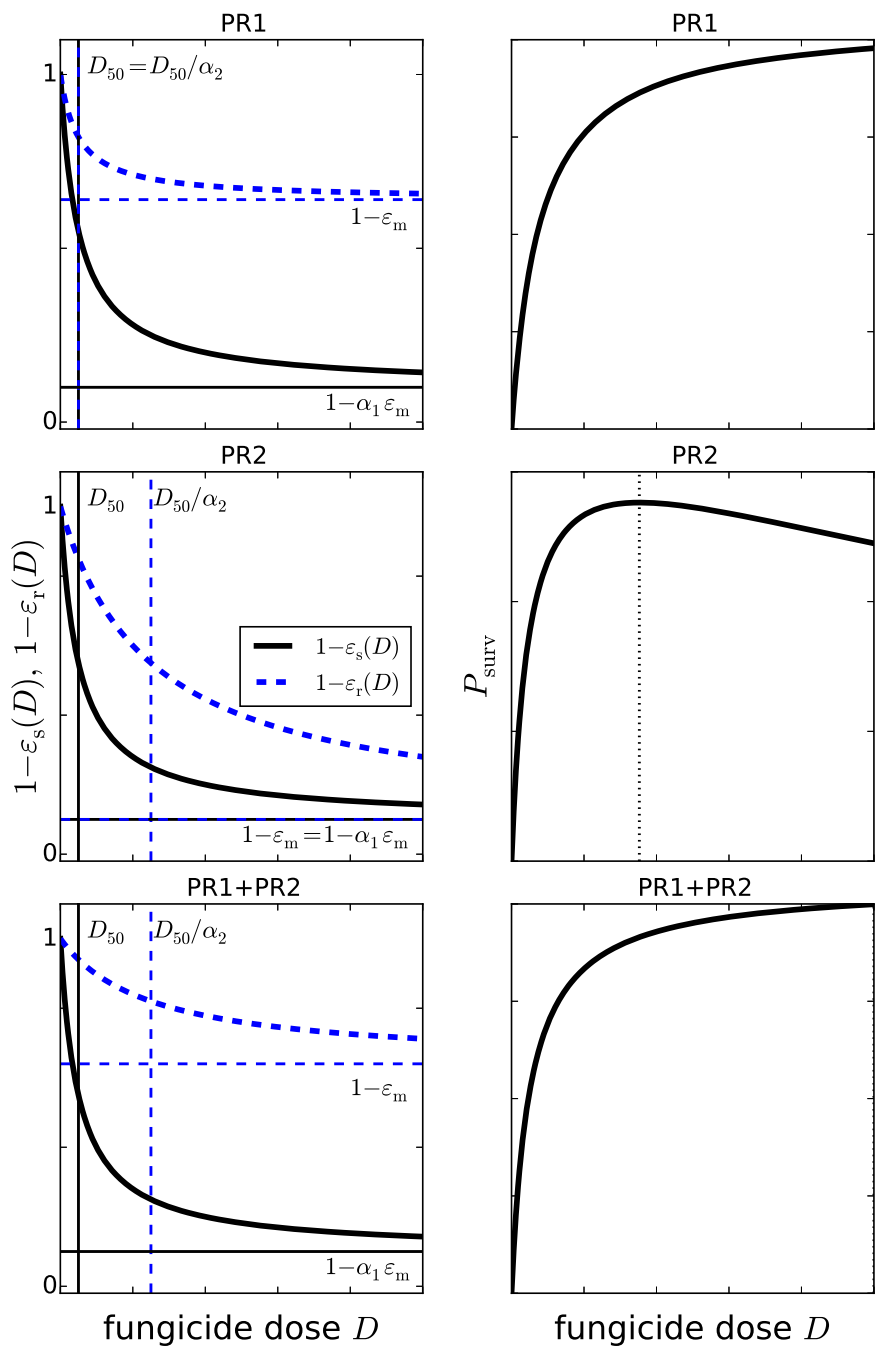

Fig. 3. Illustration of the fungicide effects, $1-\varepsilon_{s}(D)$ (solid line) and $1-\varepsilon_{r}(D)$ (dashed line), (left) and the probability of survival of a resistant mutant, $P_{\text {surv }}$ (equation 9), (right) in the case of partial resistance type 1 (PR1, $\alpha_{1}=0.4, \alpha_{2}=1$, row 1), partial resistance type 2 (PR2, $\alpha_{1}=1, \alpha_{2}=0.2$, row 2 ), and combined partial resistance of type 1 and $2\left(\mathrm{PR} 1+\mathrm{PR} 2, \alpha_{1}=0.4, \alpha_{2}=0.2\right.$, row 3$)$. 
monotonically with the dose within the range $D<1$. Therefore, for partial resistance of type 1 , the same conclusion holds as in the case of full resistance: to delay the emergence, our analysis suggests that it is better apply the lowest fungicide dose at which effective control can still be obtained.

When partial resistance affects only the shape parameter, $D_{50}$, (PR2) there can exist a dose range within the legal range of doses over which $T_{\text {emerg }}(D)$ increases with the dose (as discussed above). For this to occur, the dose at which $T_{\text {emerg }}(D)$ reaches its minimum should lie below the recommended dose, i.e., $D_{\min }<1$. Figure 6 (right column) shows that for both fungicides, the position of the minimum shifts to lower doses with increasing $\alpha_{2}$. A simple computation reveals that the minimum in $T_{\text {emerg }}(D)$ lies below the recommended dose $\left(D_{\min }<1\right)$, at $\alpha_{2}>0.2$ for tebuconazole and $\alpha_{2}>0.5$ for epoxiconazole.

When the two types of resistance (PR1 and PR2) are present together, our analysis indicates that for both tebuconazole and epoxiconazole, the minimum in $T_{\text {emerg }}(D)$ at the dose below the label recommended dose $\left(D_{\min }<1\right)$ that appears in the case of partial resistance type 2 , is vulnerable to the presence of the partial resistance of type 1: even a small decrease in the fungicide sensitivity $\alpha_{1}$ shifts $D_{\min }$ to a value larger than the label recommended dose. This makes the situation similar to the case of pure PR1 or absolute resistance: $T_{\text {emerg }}(D)$ decreases monotonically with the dose within the legal dose range.

\section{DISCUSSION}

In this study we analyzed emergence of fungicide resistance using a simple population dynamical model and compared the outcomes to empirical fungicide dose-response data recorded under field conditions. Our analysis demonstrates that time until resistance emergence, $T_{\text {emerg }}$, can (i) decrease monotonically or (ii) exhibit a minimum as a function of the fungicide dose (in the dose
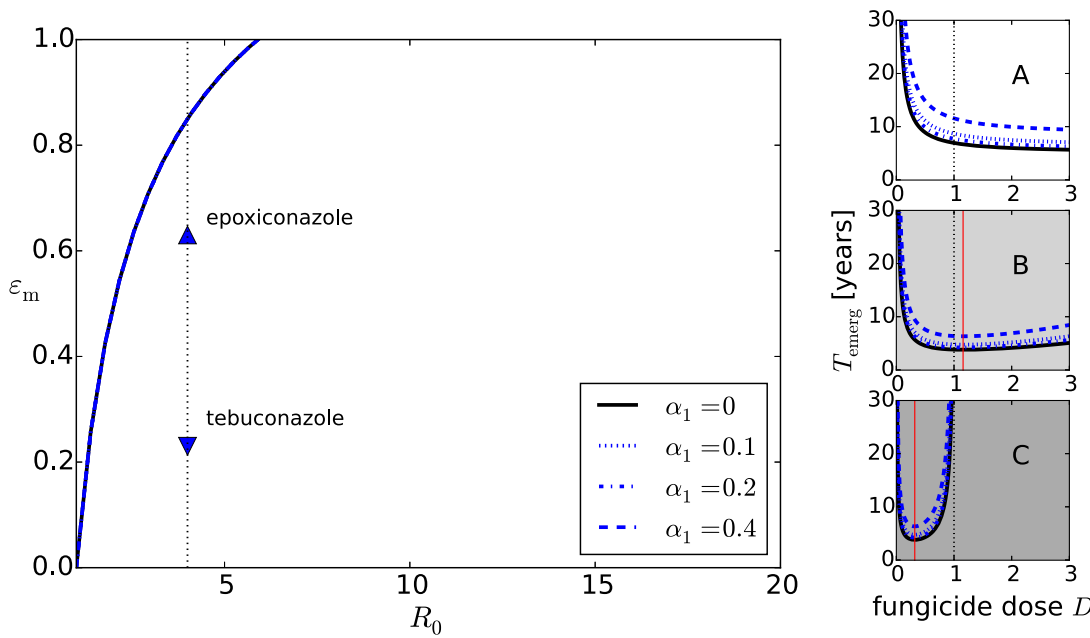

fungicide dose $D$

Fig. 4. Effect of partial resistance type 1 (PR1) on the dose-dependence of the time until emergence of fungicide resistance, $T_{\text {emerg. }}$. Here, resistance affects only $\varepsilon_{m}$, i.e., $0<\alpha_{1}<1, \alpha_{2}=1$. Left panel: Curves indicate threshold values of $\varepsilon_{m}$ and $R_{0}$ at which $D_{\min }=1$ for full resistance, $\alpha_{1}=0$ (solid line), and for PR1 at $\alpha_{1}=0.1$ (dotted line), $\alpha_{1}=0.2$ (dash-dotted line) and $\alpha_{1}=0.4$ (dashed line); all these curves are identical. The parameter region, where the dependence $T_{\text {emerg }}(D)$ exhibits a minimum at a fungicide dose $D_{\min }$ smaller than the maximum recommended dose $D_{\text {rec }}=1$, lies to the left and above the curves. The curves were obtained numerically at $D_{50}=0.7$. Right panel: The curves show $T_{\text {emerg }}$ versus the fungicide dose, $D$, for full resistance (solid line) and PR1 (dashed lines). The curves at full resistance are the same as in the right panels of Figure 1.
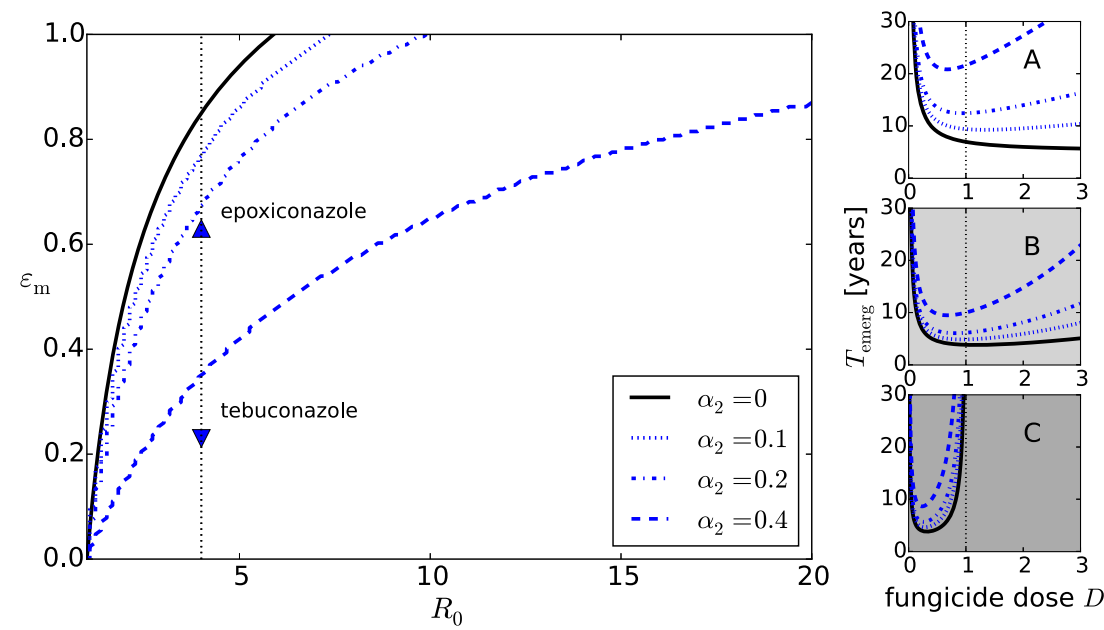

Fig. 5. Effect of partial resistance type 2 (PR2) on the dose-dependence of the time until emergence of fungicide resistance, $T_{\text {emerg }}$. Here, resistance affects only $D_{50}$, i.e., $0<\alpha_{2}<1, \alpha_{1}=1$. Left panel: Curves indicate threshold values of $\varepsilon_{m}$ and $R_{0}$ at which $D_{\min }=1$ for full resistance, $\alpha_{2}=0$ (black solid), and for PR2 at $\alpha_{2}=$ 0.1 (dotted line), $\alpha_{2}=0.2$ (dash-dotted line) and $\alpha_{2}=0.4$ (dashed line). The parameter region, where the dependence $T_{\text {emerg }}(D)$ exhibits a minimum at a fungicide dose $D_{\min }$ smaller than the maximum recommended dose $D_{m}=1$, lies to the left and above the curves. The curves were obtained numerically at $D_{50}=0.7$. Right panel: The curves show $T_{\text {emerg }}$ versus the fungicide dose, $D$, according to equation 10, for full resistance (solid line) and PR2 (dashed lines). The curves at full resistance are the same as in the right panels of Figure 1. 
range from zero up to the label recommended dose, i.e., $D_{\min }<1$ ). These two outcomes require different recommendations with regard to fungicide doses that would delay emergence of resistance: (i) use as low fungicide doses as possible at which effective control can still be obtained or (ii) use either high doses or low doses, but avoid intermediate doses.

If resistance is full or type 1 partial (resistance decreases the maximum fungicide effect), then $T_{\text {emerg }}$ decreases monotonically with dose, $D$, within the realistic range of parameter values and the legal range of doses. This is because even at high doses fungicides are typically not able to eradicate the local pathogen population, which corresponds to the relatively low values estimated for the maximum fungicide effect, $\varepsilon_{m}$ (Table 2). In this case, to delay fungicide resistance, it is recommended to use as low fungicide doses as needed for effective disease control. (For example, for foliar wheat diseases, a commonly used criterium for effective disease control is that severity is lower than $5 \%$ at the growth stage 75 , according to Zadoks scale).

If resistance is type 2 partial (resistance decreases the slope of the fungicide effect with dose), then a minimum in $T_{\text {emerg }}$ for a dose below the label recommended dose can be achieved at lower fungicide efficacies $\varepsilon_{m}$. Here, the minimum is achieved because the resistant mutants are most likely to survive in the range of intermediate doses: in this range they have the largest fitness advantage with respect to the sensitive strain (Fig. 3, middle row). However, this minimum in $T_{\text {emerg }}(D)$ easily shifts outside the legal range of doses or even disappears when the type 1 resistance is added on top of type 2 resistance (Fig. 3, third row). Thus, even in the case of PR 2 the minimum in $T_{\text {emerg }}(D)$ occurs only over a limited range of parameter values. These outcomes contrast a popular idea in the antibiotic resistance literature, that high drug doses should suppress development of partial resistance (Drlica and Zhao 2007; Lindsey et al. 2013; Read et al. 2011). Thus, outcomes of our model suggest that in the case of full resistance or PR1 resistance, resistance emerges faster at higher doses for any biologically plausible set of parameters. But for PR2 resistance, there can be cases when the emergence of resistance slows down at higher doses.

In order to provide valid advice for growers with regard to fungicide doses that would delay emergence of partial fungicide resistance, one needs to determine empirically the type partial resistance (PR1 or PR2 or both) together with the corresponding fungicide sensitivities $\alpha_{1}$ and $\alpha_{2}$. This can be done with the help of dedicated field experiments with two treatments. In the first treatment, wheat plants would be inoculated with pathogen spores of a fungicide-sensitive strain. In the second treatment, wheat plants would be inoculated with pathogen spores of a fungicide-resistant strain. Disease severity would then be measured for each of the strains as a function of the fungicide dose (dose-response curves). These experiments may require artificial inoculation with single pathogen strains, because natural infections of many fungal plant pathogens typically consist of a large number of strains (for example, Z. tritici [Linde et al. 2002]). Clearly, these measurements can only be performed in a location where a particular fungicideresistant strain have already emerged. Outcomes of these experiments would give a general idea on the range of possible values and types of resistance that are likely to emerge. More practically, the measured fungicide sensitivities $\alpha_{1}$ and $\alpha_{2}$ would help to devise a dose regime that delays emergence of resistance in geographical locations where it has not yet emerged.

In formulating the model, (i) we assumed that the fungicide-sensitive pathogen system is at equilibrium and (ii) we neglected the latent period of the pathogen. We also assumed (iii) that the fungicide dose is constant over time and (iv) that the infection develops uniformly in space, i.e., every host unit is equally likely to infect every other host unit in the population, and that fungicide application is spatially uniform. (v) We estimated both the dose-response parameters and the basic reproductive number using the data sets from a single season, single location and a single host cultivar. But these parameters can vary depending on the season, location, and cultivar. (vi) The concept of full resistance is an idealization used in the model, while in reality there may be a low level of control remaining at high doses.

We made these assumptions to keep the model simple. This allowed us to perform the analyses and gain understanding of general principles of fungicide resistance emergence over the whole range of parameter values. The emergence time-dose curve that we obtained in the case of full resistance (Fig. 1) agrees qualitatively with the curve obtained by Hobbelen et al. 2014 (Fig. 6A in Hobbelen et al. 2014), where a more biologically realistic and complex model was used without assumptions (i), (ii), and (iii) and parameterized for $Z$. tritici controlled by pyraclostrobin. This agreement indicates that in spite of its simplicity, our model captures the essential features of resistance emergence. Although, we neglected spatial effects for the sake of simplicity and tractability (assumption (iv)), they may influence emergence of fungicide resistance (Plantegenest et al. 2007). For example, intriguing and often counter-intuitive phenomena on the front of an expanding population (Hallatschek and Nelson 2009; Roques et al. 2012) may promote emergence of mutations conferring fungicide resistance. The stochastic/deterministic framework developed here can be extended using spatially explicit approaches (Lion and Gandon 2015; Mikaberidze et al. 2016; Plantegenest et al. 2007) to gain insights. With regard to assumption (v), this is the best we can do at the current state of our knowledge. Clearly, more work needs to be done to quantify the variability of the basic reproductive number with respect to the growing season, location and cultivar, the same needs to be done for the estimates of the dose-response parameters. To justify assumption (vi), we note that we use full resistance in cases when the resistance factors are so high that the resistance approximates to being 'complete' within the range of legal doses. Model dependencies of the outcomes may exist with respect to assumptions (i), (iii), and (iv). Hence, relaxing

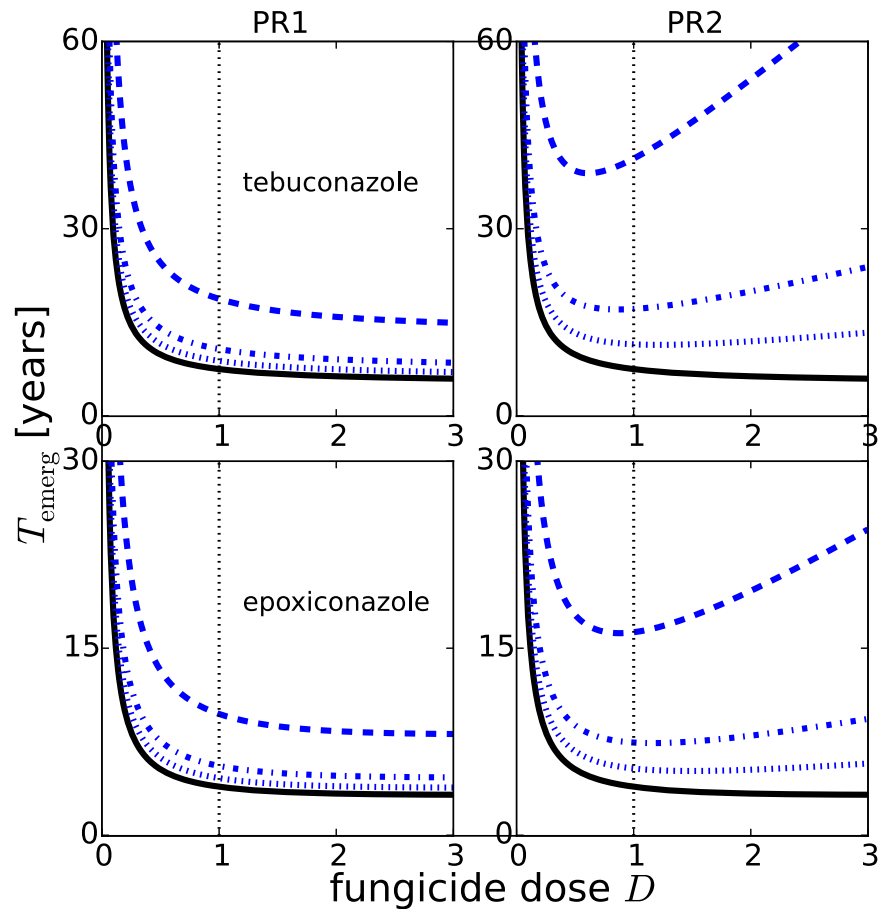

Fig. 6. Effect of partial resistance type 1 (PR1, left column) and partial resistance type 2 (PR2, right column) on the dose-dependence of the time until emergence of fungicide resistance, $T_{\text {emerg }}$, to tebuconazole and epoxiconazole. Curves show $T_{\text {emerg }}$ versus the fungicide dose, $D$, according to equation 10 . Left column, PR1: $\alpha_{1}=0.6$ (dashed line), $\alpha_{1}=0.3$ (dotted line), and $\alpha_{1}=0.15$ (dash-dotted line), $\alpha_{1}=0$ (solid line); $\alpha_{2}=1$. Right column, PR2: $\alpha_{2}=0.6$ (dashed line), $\alpha_{2}=0.3$ (dotted line) and $\alpha_{2}=0.15$ (dash-dotted line), $\alpha_{2}=$ 0 (solid line); $\alpha_{1}=1$. Upper panels use the estimates of the dose-response parameters for tebuconazole $\left(\varepsilon_{m}=0.23, D_{50}=0.5\right)$, lower panels use the estimates for epoxiconazole $\left(\varepsilon_{m}=0.63, D_{50}=0.89\right) ; C=3.8$. 
them may improve the model and lead to a more comprehensive understanding.

In the case of partial resistance, we take into account emergence of a single mutation that confers a degree of partial resistance, i.e., the first mutational step. To gain a more comprehensive understanding, emergence of several subsequent mutations needs to be considered with periods of selection in between. When the number of mutations is high, this can be achieved by combining the type of model developed here with a quantitative genetic model of plant pathogen evolution (Shaw 1989).

Here we focused on adjusting the fungicide dose as a tactic to delay emergence of fungicide resistance, but there are several other resistance management tactics available that involve two or more fungicides of different modes of action (mixtures, alternations) (van den Bosch et al. 2014). These resistance management tactics also need to be evaluated from the point of view of emergence of resistance. Nevertheless, each of these approaches involves a dose of the fungicide that is at risk of developing resistance as a parameter. Therefore, we expect our results to be useful for optimizing more complex resistance management approaches.

Fungicide resistance is a prime example of resistance of pathogens (or pests) to drugs (or biocides). The dependence of the rate of resistance development on drug dose was investigated extensively in the context of resistance of bacterial pathogens of humans to antibiotics. Kouyos et al. (2014) reviewed outcomes of 51 empirical studies on the effect of drug dose on development of antimicrobial resistance for a range of human pathogens. However, the effect of dose on the emergence phase could not be clearly separated from the effect on the selection phase of resistance development. The majority of in vitro studies indicated that aggressive chemotherapy (high doses) is better at suppressing development of resistance. In vivo results in animal models and in humans were less clear: 14 out of 33 studies favored aggressive treatment, eight studies indicated moderate treatment (low doses) is advantageous, and five studies reported mixed or neutral evidence. This marked difference between in vitro and in vivo studies may result from the effect of the host immune system or from differential drug penetration in the host tissues.

In contrast, our results indicate that higher doses are likely to accelerate emergence of fungicide resistance due to relatively low efficacy of fungicides under field conditions. Even at high fungicide doses close to the maximum recommended dose, disease severity often remains significant, at levels 5 to $10 \%$ (Lockley and Clark 2005; Paveley et al. 1998) for dose-response data for several important foliar fungal pathogens of wheat including Z. tritici, Parastagonospora nodorum (formerly Septoria nodorum), and Blumeria graminis and many fungicides including strobilurins and azoles). Therefore, although the pathogen population may be suppressed below the economic damage threshold, the fungicide-sensitive subpopulation is still large enough such that the rate of appearance of resistant mutants is not a limiting factor for emergence of resistance. We estimated the maximum decrease, $\varepsilon_{m}$, in $Z$. tritici's basic reproductive number, $R_{0}$, due to fungicide action to be in the range of 30 to $60 \%$ (Table 2). This decrease may suppress the development of a severe epidemic, but is unlikely to drive the local population of this pathogen to extinction. Possible reasons for this relatively mild effect of fungicides include imperfect coverage of plant tissue by fungicide sprays and reservoir of the pathogen spores and mycelial particles in the environment. This is in stark contrast to the situation in the in vitro populations of bacterial human pathogens subjected to antibiotics. Antibiotics are quite effective at killing bacteria or suppressing their reproduction and thus can prevent the appearance of resistant mutants through mutation at high enough doses. This difference also reflects difference between the target of antibiotic therapy in humans (to eradicate the pathogen in the host) and the target of fungicide treatments of crops (to drive the pathogen population below the damage threshold).

It appears that in medical literature, ideas that aggressive chemotherapy helps to suppress development of resistance are largely based on in vitro studies (Kouyos et al. 2014; Roberts et al. 2008), although there is also a number of counter-examples (Day and Read 2016). When both in vitro and in vivo results are considered together, the outcomes do not show a consistent pattern (Day and Read 2016; Kouyos et al. 2014). General usefulness of aggressive chemotherapy as a way to suppress the development of drug resistance was criticized in several recent studies (Day and Read 2016; Huijben et al. 2013; Kouyos et al. 2014; Read et al. 2011). Similarly, in the context of crop protection, some ideas on using high doses to reducing the risk of resistance seem to have come from insecticide resistance literature, where insect pests are typically diploid and reproduce sexually. In this case, high doses are likely to delay resistance emergence by removing the majority of resistance genes from the population, as they are initially contained within heterozygote individuals (provided the resistance is sufficiently recessive). This reasoning does not apply to most fungal plant pathogens, which are either haploid or clonal (van den Bosch et al. 2011).

\section{APPENDIX A. MODEL OF EMERGENCE OF FUNGICIDE-RESISTANT MUTANTS}

Consider dynamics of the healthy host density, $H$, and host density infected by the sensitive pathogen strain, $I_{s}$, governed by the system of ordinary differential equations

$$
\begin{gathered}
\frac{d H}{d t}=r_{H}\left(K-H-I_{s}\right)-\beta_{s} I_{s} H \\
\frac{d I_{s}}{d t}=\beta_{s} I_{s} H-\mu I_{s}+\delta \\
\frac{d R}{d t}=\mu I_{s}
\end{gathered}
$$

where $R$ is the density of hosts infected by the sensitive strain, which is no longer infectious (i.e., the "removed" compartment). The number of lesions produced by the resistant pathogen strain is described by a time-inhomogeneous birth-death process with the birth rate

$$
B(t)=\beta_{r} H(t)
$$

and the death rate $\mu$. Using the formalism of time-inhomogeneous Markov chains, we determine the probability of extinction of a single mutant after time $T$, given that it exists at time $t$ :

$$
p_{\text {ext }}(T)=\operatorname{Pr}\left(Y_{r}(t+T)=0 \mid Y_{r}(t)=1\right)=\frac{\mu \int_{t}^{t+T} e^{R(\tau)} d \tau}{1+\mu \int_{t}^{t+T} e^{R(\tau)} d \tau}
$$

where $R(\tau)=\mu(\tau-t)-\int_{t}^{\tau} B\left(t^{\prime}\right) d t^{\prime}$. The probability of ultimate survival of a single mutant that existed at time $t$ is calculated using equation 18 by taking the limit $T \rightarrow \infty$ :

$$
P_{\text {surv }}(t)=1-p_{\text {ext }}(T \rightarrow \infty)=\frac{1}{1+\mu \int_{t}^{\infty} \exp \left[\mu(\tau-t)-\beta_{r} \int_{t}^{\tau} H\left(t^{\prime}\right) d t^{\prime}\right] d \tau}
$$

Here, we substituted equation 17 for the birth rate.

The probability of appearance of a resistant mutant per unit time is given by

$$
r_{\text {app }}(t)=\frac{m \beta_{s} o}{\psi} I_{s}(t) H(t)
$$

where $m$ is the probability of mutation from the fungicide-sensitive pathogen type to the fungicide-resistant pathogen type, $o$ is the land 
area occupied by host plants, and $\psi$ is the leaf area of a single lesion. The factor $o / \psi$ in equation 8 converts the density, $I_{s}$, used in the deterministic part of the model, to the number of individuals (counted as lesions) used in the stochastic part. Here, a transmission event is required for a resistant individual to appear, and hence the rate of appearance of mutants is proportional to the transmission rate, $\beta_{s}$, and the healthy host density.

The probability per unit time of resistance emergence (rate of emergence) is given by

$$
\rho(t)=r_{\text {app }}(t) P_{\text {surv }}(t)
$$

The waiting time $T$ until the first appearance of a resistant mutant is the time until the first event in a nonhomogeneous Poisson process with the rate $\rho(t)$ occurs. Then, the distribution of $T$ can be written in the form of a cumulative density function (CDF)

$$
F_{T}(t)=\operatorname{Pr}(T \leq t)=1-\operatorname{Pr}(X(t)=0)=1-e^{-\int_{0}^{t} \rho(\tau) d \tau}
$$

where $X(t)$ is the number of events that occurred by time $t . F_{T}(t)$ in equation 22 is the probability that at least one resistant mutant emerges until time $t$. We obtain the ultimate emergence probability from equation 22 by taking the limit $t \rightarrow \infty$

$$
P_{\text {emerg }}=1-e^{-\int_{0}^{\infty} \rho(\tau) d \tau}
$$

From the $\operatorname{CDF} F_{T}(t)$ in equation 22 , we obtain the probability density function (PDF) by differentiating it with respect to $t$ :

$$
f_{T}(t)=\operatorname{Pr}(T \leq t)=\rho(t) e^{-\int_{0}^{t} \rho(\tau) d \tau}
$$

We then determine the mean waiting time, i.e., mean time until emergence of fungicide resistance by calculating the first moment of the PDF in equation 24:

$$
T_{\text {emerg }}=\int_{0}^{\infty} t f_{T}(t) d t
$$

The outcomes of host-pathogen dynamics governed by equations 14,15 , and 16 are determined by the parameter combination

$$
R_{0 s}=\frac{\beta_{s} K}{\mu}
$$

that is called the basic reproductive number (Anderson and May 1986). If $R_{0 s}>1$, then the sensitive pathogen strain survives and eventually reaches endemic equilibrium with the host population, where

$$
H(t)=H^{*}=\mu / \beta_{s}, I_{s}(t)=I_{s}^{*}=\frac{r_{H}\left(\beta_{s} K-\mu\right)}{\beta_{s}\left(\mu+r_{H}\right)}
$$

On the other hand, if $R_{0 s}<1$, then the sensitive pathogen strain dies out and host densities $H(t)$ and $I_{s}(t)$ reach the values corresponding to the uninfected equilibrium:

$$
H(t)=H^{*}=K, I_{s}(t)=I_{s}^{*}=0
$$

We substitute equation 27 in equation 17 and obtain the following expression for the birth rate at endemic equilibrium

$$
B=\mu \frac{1-\theta}{1-\varepsilon_{s}(D)}
$$

Next, we determine the probability of ultimate survival of a single mutant at endemic equilibrium by substituting $H(t)=H^{*}$ in equation 19 , where $H^{*}$ is given by equation 27 or equation 28

$$
P_{\text {surv }}=1-p_{\text {ext }}(T \rightarrow \infty)=1-\frac{\mu}{\beta_{r} H^{*}}= \begin{cases}1-1 / R_{0 r}, & \text { if } R_{0 s}<1 \\ 1-R_{0 s} / R_{0 r}, & \text { if } R_{0 s}>1\end{cases}
$$

where we introduced the basic reproductive number of the resistant strain

$$
R_{0 r}=\frac{\beta_{r} K}{\mu}
$$

In the case of full resistance considered here, the survival probability $P_{\text {surv }}$ depends on the fungicide dose only if $R_{0 s}>1$. We determine this dependence explicitly by substituting for the transmission rates $\beta_{r}=\beta(1-\theta)$ and $\beta_{s}=\beta\left[1-\varepsilon_{s}(D)\right]$ in equation 30:

$$
P_{\text {surv }}=\frac{\varepsilon_{s}(D)-\theta}{1-\theta}
$$

This quantity increases monotonically with the fungicide dose $D$ and decreases monotonically with the fitness $\operatorname{cost} \theta$. Interestingly, if resistance does not confer a fitness cost, i.e., $\theta=0$, then the probability of survival is always nonzero. However, if there is a fitness cost, then $P_{\text {surv }}$ equals zero at $\theta \geq \varepsilon_{s}(D)$.

The rate of appearance of resistant mutants at endemic equilibrium is obtained by substituting equation 27 in equation 20

$$
r_{a p p}=\frac{1}{C} \frac{(1-\theta)\left(R_{0}\left[1-\varepsilon_{s}(D)\right]-1\right)}{\left[1-\varepsilon_{s}(D)\right]}
$$

where

$$
R_{0}=\frac{\beta K}{\mu}
$$

is the basic reproductive number of the pathogen without fungicides or resistance fitness costs and

$$
C=\frac{\beta \psi\left(\mu+r_{H}\right)}{\operatorname{mor}_{H} \mu^{2}}
$$

Then, the rate of resistance emergence is given by

$$
\rho=r_{\text {app }} P_{\text {surv }}=\frac{1}{C} \frac{\left(R_{0}\left[1-\varepsilon_{s}(D)\right]-1\right)\left[\varepsilon_{s}(D)-\theta\right]}{\left[1-\varepsilon_{s}(D)\right]}
$$

Since the rate $\rho$ is time-independent here, the ultimate emergence probability $P_{\text {emerg }}$ in equation 23 is

$$
P_{\text {emerg }}= \begin{cases}1, & \text { if } \rho>0 \\ 0, & \text { if } \rho=0\end{cases}
$$

The mean waiting time until emergence of resistance (equation 25) makes sense only when resistance will certainly emerge, i.e., at $P_{\text {emerg }}=1$. In this case, the mean waiting time simply yields, according to equation 25 ,

$$
T_{\text {emerg }}=1 / \rho=C \frac{\left[1-\varepsilon_{s}(D)\right]}{\left(R_{0}\left[1-\varepsilon_{s}(D)\right]-1\right)\left[\varepsilon_{s}(D)-\theta\right]}
$$

We determined two critical fungicide doses. The first one $D_{c 1}$ exists when there is a fitness cost to resistance, i.e., $\theta>0$. In this case, at low doses when $D<D_{c 1}$, many resistant mutants appear per unit time, but they lose competition with the already existing sensitive pathogen strain, because of the fitness cost. At $D=D_{c 1}$, the disadvantage for the sensitive strain due to the fungicide is exactly compensated by the disadvantage for the resistant strains due to the fitness cost, such that $R_{0 s}=R_{0 r}$. From $R_{0 s}=\beta\left(1-\varepsilon_{s}(D)\right) K / \mu$ and $R_{0 r}=$ $\beta(1-\theta) K / \mu$, we find $D_{c 1}$ by solving the equation $R_{0 s}=R_{0 r}$ with respect to $D$, taking $\varepsilon_{s}(D)=\varepsilon_{m} D /\left(D+D_{50}\right)$ : 


$$
D_{c 1}=D_{50} \theta /\left(\varepsilon_{m}-\theta\right)
$$

Second critical dose, $D_{c 2}$, corresponds to the threshold dose above which the sensitive strain is eliminated, i.e., $R_{0 s}=1$ :

$$
D_{c 2}=D_{50}\left(R_{0}-1\right) /\left(1-R_{0}\left(1-\varepsilon_{m}\right)\right)
$$

\section{APPENDIX B. DEPENDENCE OF THE MEAN TIME UNTIL EMERGENCE ON THE FUNGICIDE DOSE: THREE REGIMES}

In this section, we analyze the expression in equation 10 for the mean time until emergence of fungicide resistance, $T_{\text {emerg }}(D)$, as a function of the fungicide dose, $D$. More specifically, we would like to find out under which conditions the function $T_{\text {emerg }}(D)$ exhibits a minimum, and under which conditions it manifests a monotonic decline.

We solve the equation $d T_{\text {emerg }} / d t=0$ with respect to the fungicide dose $D$, in order to determine the range of parameter values over which the function $T_{\text {emerg }}(D)$ has an extremum (we are interested in a minimum) and the corresponding fungicide dose $D_{\min }$ expressed in terms of other parameters. The solution yields the expression

$$
D_{\text {min }}=D_{50} \frac{R_{0}\left(1-\varepsilon_{m}\right)-1+\varepsilon_{m} \sqrt{R_{0}(1-\theta)}+\theta}{1+R_{0}\left(1-\varepsilon_{m}\right)^{2}-\theta}
$$

assuming that the dose-response curve $\varepsilon_{s}(D)$ follows the Hill function (equation 4 in the main text). The minimum is relevant only when it appears in the relevant range of fungicide doses, i.e., $D_{c 1}<$ $D<D_{\text {rec }}$, where $D_{c 1}$ is given by equation 39 and $D_{r e c}$ is the label recommended dose, which we set to be a unit dose: $D_{\text {rec }}=1$.

The condition $D_{\min }>D_{c 1}$ is equivalent to

$$
\varepsilon_{m}>\varepsilon_{m c 1}=1-\sqrt{(1-\theta) / R_{0}}
$$

The condition 42 means that in order for the $T_{\text {emerg }}(D)$ to exhibit a minimum, the maximum efficacy of the fungicide $\varepsilon_{m}$ must exceed a

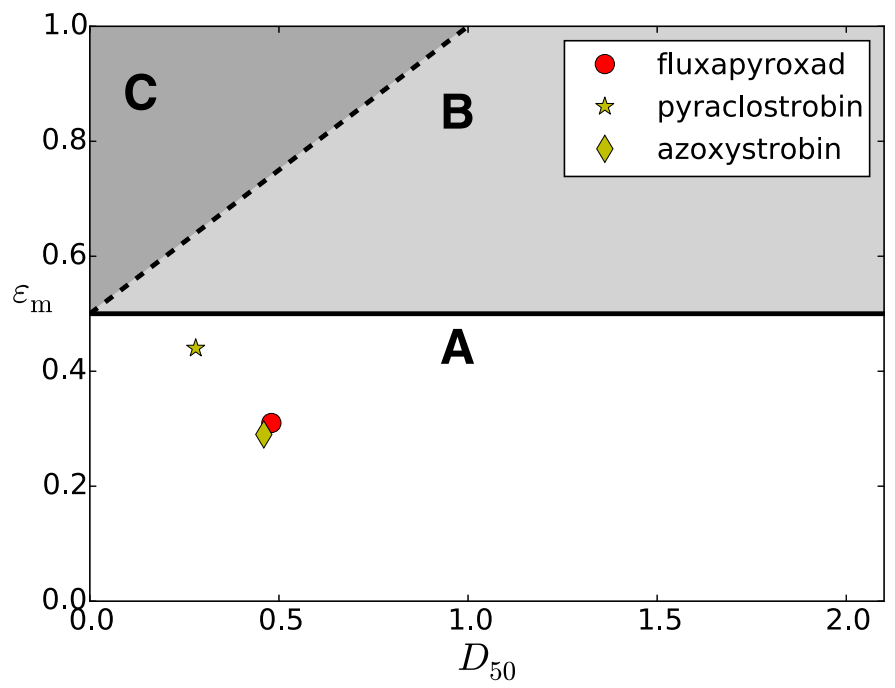

Fig. 7. The three regimes of the dependence of $T_{\text {emerg }}$ on the fungicide dose, $D: \mathrm{A}$ (white area), B (light gray area), and C (dark gray area) (see also text and Fig. 1) versus the maximum fungicide effect, $\varepsilon_{m}$, and the shape parameter, $D_{50}$. Solid line corresponds to $\varepsilon_{m c 1}$ and is plotted according to equation 12; dashed line corresponds to $\varepsilon_{m c 2}$ and is plotted according to equation 13 at $R_{0}=4$ (solid curve), the estimate for Zymoseptoria tritici. The points indicate the best-fit values of $D_{50}$ and $\varepsilon_{m}$ for the three fungicides (fluxapyroxad, pyraclostrobin, azoxystrobin) (Table 2). We considered full fungicide resistance and no immigration $\left(\delta_{s}=0\right)$. critical value $\varepsilon_{m c 1}$ that depends on the pathogen's basic reproductive number, $R_{0}$, and the fitness cost, $\theta$, associated with the resistance mutation.

If there is no fitness cost of resistance, the condition for having a minimum in $T_{\text {emerg }}(D)$ reads (here we substituted $\theta=0$ in equation 42)

$$
\varepsilon_{m}>\varepsilon_{m c 1}=1-\frac{1}{\sqrt{R_{0}}}
$$

and is shown using a solid curve in Figure 1.

When the condition of equation 42 is fulfilled, there is a minimum in $T_{\text {emerg }}(D)$. Hence, there is a range of doses over which $T_{\text {emerg }}(D)$ increases with dose. However, this range can be reached in practice only if $D_{\min }$ is smaller than the label recommended dose, i.e., $D_{\min }<$ $D_{\text {rec }}=1$. Substituting equation 11 in the inequality $D_{\text {min }}<1$, we find

$$
\varepsilon_{m}>\varepsilon_{m c 2}=\left(D_{50}+1\right)\left(1-\sqrt{(1-\theta) / R_{0}}\right)
$$

The inequality equation 44 poses a more stringent requirement on $\varepsilon_{m}$ than the inequality equation 42 , since $\varepsilon_{m c 2} \geq \varepsilon_{m c 1}$. Figure 1 shows $\varepsilon_{m c 2}$ as a function of the basic reproductive number, $R_{0}$ using a dashed curve. $\varepsilon_{m c 2}$ is proportional to $D_{50}$ and, therefore, the condition (44) also limits the range of the values of $D_{50}$. This is illustrated in Figure 7, where $\varepsilon_{m c 2}$ is shown as a function of $D_{50}$ at $R_{0}=4$ (the value estimated for $Z$. tritici, Appendix $C$ ). The two dose-response parameters play different roles. The maximum fungicide effect, $\varepsilon_{m}$ determines both the appearance of the minimum in $T_{\text {emerg }}(D)$ and the position of the minimum. In contrast, $D_{50}$ does not influence the appearance of the minimum, but only affects its position.

Note also that $\varepsilon_{m c 2}$ as a function of the basic reproductive number, $R_{0}$, reaches its maximum value of unity at $R_{0}=\mathrm{R}_{0 b}$, where

$$
R_{0 b}=\frac{D_{50}+2-2 \theta\left(D_{50}+1\right)}{D_{50}(1-\theta)}
$$

When $R_{0}$ exceeds this value, then fungicide resistance will emerge faster at larger doses at any values of $\varepsilon_{m}$.

If the fungicide is effective enough and the pathogen's basic reproductive number, $R_{0}$, is low enough, the sensitive pathogen subpopulation can be eliminated at fungicide doses that exceed $D_{c 2}$ (equation 40). The inequality $D_{c 2}>0$ provides us with the range of values of $\varepsilon_{m}$ and $R_{0}$ over which this effect is realized.

$$
\varepsilon_{m}>\varepsilon_{m c 3}=1-1 / R_{0}
$$

In this regime, the rate of emergence vanishes, i.e., $\rho=0$.

\section{APPENDIX C. PARAMETER VALUES}

We first estimate the basic reproductive number of $Z$. tritici using the estimate of the exponential growth rate. Then, we describe the choice of values for other life-cycle parameters of this pathogen. Finally, we estimate the fungicide dose-response parameters by fitting the dose-response curves.

Relationship between the exponential growth rate of an epidemic with the basic reproductive number. When a healthylatent-infected-removed (H-L-I-R) epidemiological model is used to describe epidemics of plant diseases the exponential growth rate at the beginning of the epidemic is connected to the basic reproductive number, $R_{0}$, mean latent period $\Delta t_{l}$ and mean infectious period $\Delta t_{i}$ in the following way (Madden et al. 2006a):

$$
r_{e}=\sqrt{\mathrm{u}^{2}+\frac{R_{0}-1}{\Delta t_{l} \Delta t_{i}}}-\mathrm{u}
$$

where $u=\left(\Delta t_{l}+\Delta t_{i}\right) /\left(2 \Delta t_{l} \Delta t_{i}\right)$. On the other hand, when using a simpler healthy-infected-removed (H-I-R) model without a latent 
compartment, the relationship between the exponential growth rate and the basic reproductive number becomes simpler:

$$
r_{e}=\left(R_{0}^{*}-1\right) / \Delta t_{g}
$$

where $t_{g}$ is the mean generation time and $R_{0}^{*}$ is the basic reproductive number in the H-I-R model. We establish a relationship between $R_{0}$ and $R_{0}^{*}$ by equating $r_{e}$ in equation 47 and equation 48, and setting $\Delta t_{g}=\Delta t_{i}+\Delta_{l}$ :

$$
R_{0}^{*}=1-\mathrm{u}\left(\Delta t_{i}+\Delta t_{l}\right)+\sqrt{\frac{R_{0}-1}{\Delta t_{i} \Delta t_{l}}+\mathrm{u}^{2}}
$$

We take an example of $Z$. tritici on wheat, where the estimate $r_{e}=$ 0.1173 was obtained by fitting the disease progress curve in the beginning of an epidemic to an exponential function (P. Hobbelen, personal communication). The latent period was taken to be $\Delta t_{l}=$ 16 days (same value as in Hobbelen et al. 2011), infectious period $\Delta t_{l}=10$ days (Eyal 1971). In this case, $R_{0}=6.2$ is found by solving equation 47 with respect to $R_{0}$ and $R_{0}^{*}=4.0$ is found by solving equation 48 with respect to $R_{0}^{*}$. Similar values are obtained from averaging $R_{0}$ over 42 data cases when fitting the DPC of $Z$. tritici from IDR4 experiments to a simple population dynamical model (A. Mikaberidze, N. Paveley, F. van den Bosch, F. van den Berg, and $\mathrm{S}$. Bonhoeffer, unpublished data). H-L-I-R is a more realistic model to describe epidemics of Z. tritici on wheat, but we use the H-I-R model for the sake of simplicity. We use the value $R_{0}^{*}=4.0$ for the basic reproductive number that gives the same exponential growth rate as in H-L-I-R model. However, the correct epidemic threshold should be obtained using the basic reproductive number from the $\mathrm{H}$ L-I-R model, i.e., $R_{0}=6.2$.

Life-cycle parameters of $\boldsymbol{Z}$. tritici. We estimate the rate of appearance constant $C=\beta o\left(\mu+r_{H}\right) /\left(m \psi r_{H} \mu^{2}\right)$ by setting the time until emergence for azoxystrobin at the label recommended dose $(D=1)$ to 7 years. This is reported to be the average time from the introduction of QoI fungicides on the market until the detection of resistant strains in the UK (Grimmer et al. 2014). From this, we find $\mathrm{C}=3.8$. Without pathogen immigration, the rate of emergence of resistance, $\rho(D)$, depends on the parameters $\mu, r_{H}$, and $m$, only through their combination in the appearance constant, $C$. Hence, we do not need to estimate these parameters separately. But when we include pathogen immigration (subsection "Pathogen immigration" of the "Results" section in the main text), $\rho(D)$ depends explicitely on each of these parameters (equation 56). Therefore, when plotting Figure 2, we used specific values for the parameters $\mu, r_{H}, K, o$, and $\psi$. The effective rate of infectivity loss was estimated $\mu=1 /\left(\Delta t_{l}+\right.$ $\left.\Delta t_{i}\right)=1 / 26$ days $^{-1}$, where we used $\Delta t_{l}=16$ days (the same as in Hobbelen et al. 2011) and $\Delta t_{l}=10$ days (obtained using the data recorded by Eyal 1971) (Fig. 8). The values of the growth rate of healthy hosts and the carrying capacity were set as $r_{H}=0.2$ and $K=4$ (same as in Hobbelen et al. 2014). These values are close to the values we obtained from fitting a large number of disease progress curves of Z. tritici recorded in the U.K. to the population dynamical model similar to equation 1 and equation 2 (A. Mikaberidze, N. Paveley, F. van den Bosch, F. van den Berg, and S. Bonhoeffer, unpublished data). As Hobbelen et al. (2014), we set the size of the host plants growing area to $o=350,000 \mathrm{~km}^{2}$, which corresponds to the winter wheat growing area in Europe during the years 2000

TABLE 2. Fungicide dose-response parameters for controlling Zymoseptoria tritici on wheat

\begin{tabular}{lcc}
\hline Fungicide & $D_{50}$ & $\varepsilon_{m}$ \\
\hline Fluxapyroxad & $0.50 \pm 0.20$ & $0.31 \pm 0.08$ \\
Tebuconazole & $0.50 \pm 0.05$ & $0.23 \pm 0.01$ \\
Epoxiconazole & $0.90 \pm 0.10$ & $0.63 \pm 0.05$ \\
Pyraclostrobin & $0.28 \pm 0.06$ & $0.44 \pm 0.03$ \\
Azoxystrobin & $0.46 \pm 0.04$ & $0.29 \pm 0.01$ \\
\hline
\end{tabular}

to 2008 (Eurostat, the statistical office of the European Union). Lesion size was set $\psi=0.3 \mathrm{~cm}^{2}$, as in Robert et al. (2008).

We note, that the mutation probability, $m$, acts merely as a scaling factor in the emergence time-dose curves and its value does not affect any of our qualitative conclusions. Nevertheless, we will make a rough estimate of the mutation probability, $m$, by setting $C=$ $\beta o\left(\mu+r_{H}\right) /\left(m \psi r_{H} \mu^{2}\right)=3.8$ and using the parameter values above: $m=1.9 \times 10^{-18}$. The number of single nucleotide substitutions per nucleotide per generation, $u$, is proportional to the mutation probability, $m$, with a factor $\beta / u$ (hence, $u=\psi\left(\mu+r_{H}\right) /\left(\operatorname{Cor}_{H} \mu^{2}\right)$. Hence, we estimate, $u=1.9 \times 10^{-18}$. A typical genome-wide substitution rate for eucaryotes, (including fungi) is around $10^{-8}$ per nucleotide per generation. Possible reasons why our estimate is much lower include: (i) it is possible that a second, enabling mutation must occur simultaneously with the G143A mutation in cytochrome b gene to confer high levels of QoI resistance in Z. tritici (see for example, Miguez et al. (2004)) (ii) in the presence of many mitochondria in a cell and therefore many copies of the mitochondrial DNA, a sufficiently large number of them must carry a resistance mutation (i.e., G143A) in order for the resistance phenotype to manifest. For this reason, the rate at which resistant individuals arise through mutation can be much lower than the rate of single nucleotide substitution.

Fitting dose-response curves. In this section, we present details on fitting the dose-response curves for five fungicides: fluxapyroxad, tebuconazole, epoxiconazole, pyraclostrobin, and azoxystrobin. The fungicides were applied to control $Z$. tritici on wheat. To estimate the dose-response parameters $\varepsilon_{m}$ and $D_{50}$ in the model (equation 4), we need to determine the relationship between the decrease in the transmission rate $\beta_{s}$ and the decrease in the disease severity, $y$, defined as

$$
y(t)=\frac{I_{s}(t)+R(t)}{I_{s}(t)+R(t)+H(t)}
$$

We assume that during the period between the time of fungicide application $t=t_{0}$ and the time of severity assessment $t=t_{1}$, the host density in the infected compartment grows exponentially, i.e.,

$$
I_{s}=I_{s 0} \exp [r(D) t]
$$

where the growth rate, $r(D)$, depends on the fungicide dose $D . r(D)$ is related to other model parameters in the following manner

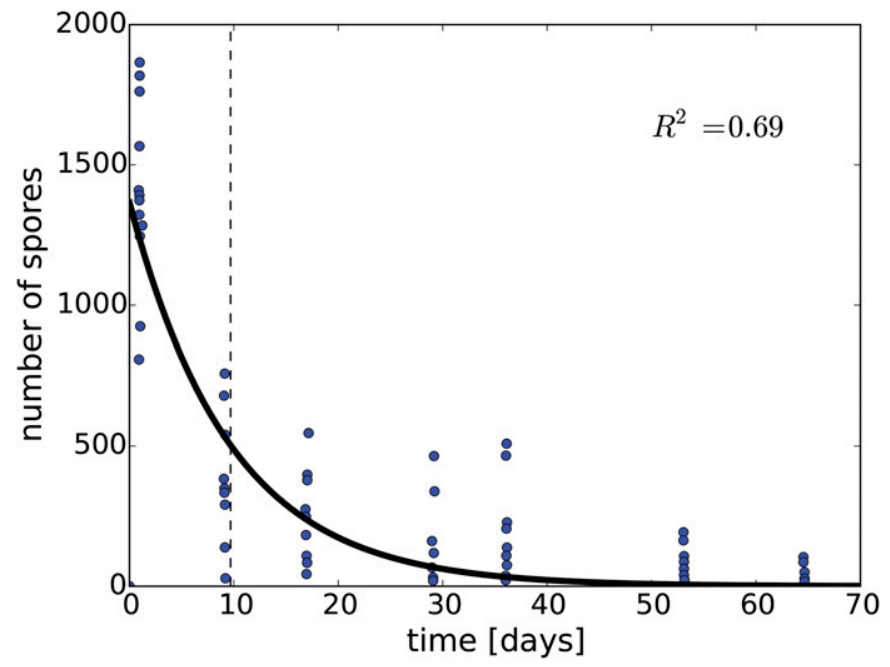

Fig. 8. Number of spores naturally exuded versus time. The data points were taken from Figure 1 in Eyal (1971) and fitted using the exponential function $y=y_{0} \exp (-\mu t)$. The best-fit values: $y_{0}=1,400,1 / \Delta t_{i}=0.10$. 


$$
r(D)=\beta\left[1-\varepsilon_{s}(D)\right] K-\mu=\mu\left(R_{0}\left[1-\varepsilon_{s}(D)\right]-1\right)
$$

where $R_{0}=\beta K / \mu$ and

$$
\varepsilon_{s}(D)=\varepsilon_{m} \frac{D^{\kappa}}{D^{\kappa}+D_{50}^{\kappa}}
$$

Next, we substitute equation 51 in equation 16 and perform integration with respect to time $t$ with the initial condition $R(t=0)=$ 0 , to obtain

$$
I_{s}+R=I_{s 0}[(1+\mu / r(D)) \exp [r(D) t]-\mu / r(D)]
$$

The assumption of exponential growth of $I_{s}(t)$ implies in addition that $I_{s}+R+H \approx K$. Consequently, we obtain the expression for the disease severity, neglecting the dependence of the coefficient before the exponential function on the fungicide dose $D$ :

$$
y(t)=y_{0} \exp \left[\left(R_{0}\left[1-\varepsilon_{s}(D)\right]-1\right) t\right]
$$

where we neglected the term $I_{0} \mu / r(D)$, which is much smaller than the exponentially growing term.

We set the time of fungicide application to zero $\left(t_{0}=0\right)$ and fit the dependence of the disease severity at the time of assessment, $t_{1}$, to the field data with three fitting parameters: $y_{0}, \varepsilon_{m}$, and $D_{50}$. We use $\kappa=1$, set $t_{1}=30$ days, $R_{0}=4.0, \mu=1 / 26$ day $^{-1}$. In this way, we fitted the dose-response curves for succinate dehydrogenase inhibitor (SDHI) fungicides (fluxapyroxad in Figure 9), two azoles (tebuconazole in Figure 10 and epoxiconazole in Figure 11 [Paveley et al. 1998]), and two strobilurines (pyraclostrobin in Figure 12 and azoxystrobin in Figure 13).

We assumed an absolute uncertainty in severity measurements to be $10 \%$ and determined $95 \%$ confidence intervals of the parameter estimates from standard errors. Relatively small confidence intervals compared with the estimate values suggest that the parameters are identifiable. The standard errors of the regression are small compared with the values of disease severity and $R^{2}$ values are close to one, indicating successful fits.

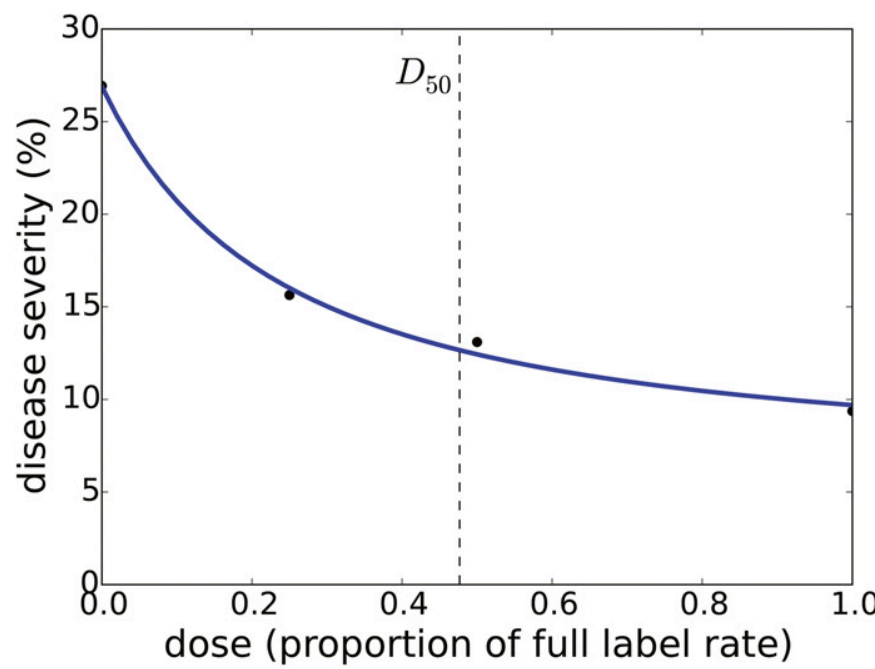

Fig. 9. Severity of Zymoseptoria tritici as a function of the fluxapyroxad (Imtrex) dose. Points are the results of field experiments reported in (www.hgca.com/media/302481/wheat-fungicide-performance-data-2013. pdf, accessed on 28 March 2016) averaged over protectant and eradicant doseresponse curves for 2012 to 2013 . The curve represents the best fit to the data points assuming exponential increase of disease severity (according to equation 55) and that the transmission rate $\beta$ decreases with the dose according to the Hill function. The best-fit parameter values: $D_{50}=0.5 \pm 0.2, \varepsilon_{m}=0.31 \pm$ $0.08, y_{0}=(7.0 \pm 0.3) \times 10^{-3}$. The value of the basic reproductive number without fungicide was taken to be $R_{0}=4.0, t_{1}=31.5$ days, $\mu=1 / 26$ day $^{-1}$. The quality of fit: standard error of regression $s=8.3 \times 10^{-3}, R^{2}=0.996$.
The measurements of the dose-response curves characterized both the protectant and eradicant action of fungicides. To evaluate the protectant action, the fungicide was applied on wheat leaves as soon as they emerged, before any visible disease symptoms appeared. To assess the eradicant action, the fungicide was applied after the disease symptoms became visible. The results for fluxapyroxad were obtained in 2012 and 2013 and averaged over 12 trials for protectant data and over five trials for eradicant data [data obtained from https://cereals.ahdb.org.uk/media/302481/ wheat-fungicide-performance-data-2013.pdf]. Here, we took the mean of protectant and eradicant dose-response curves.

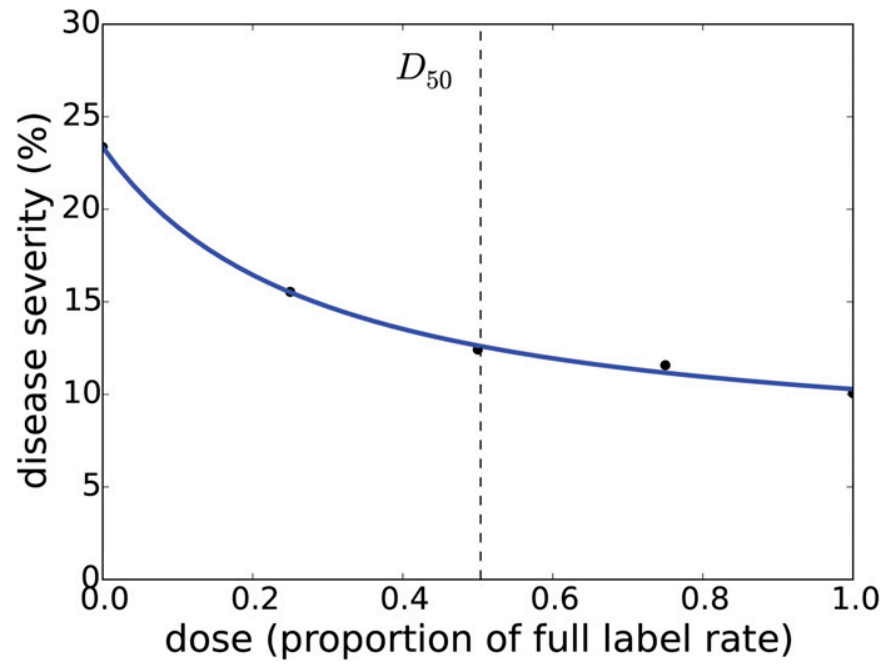

Fig. 10. Severity of Zymoseptoria tritici as a function of the tebuconazole (Folicur) dose. Points are the results of field experiments reported in (Paveley et al. 1998). The curve represents the best fit to the data points assuming exponential increase of disease severity (according to equation 55) and that the transmission rate $\beta$ decreases with the dose according to the Hill function. The best-fit parameter values: $D_{50}=0.50 \pm 0.05, \varepsilon_{m}=0.23 \pm 0.01, y_{0}=(4.12 \pm$ $0.03) \times 10^{-3}$. The value of the basic reproductive number without fungicide was taken to be $R_{0}=4.0, t_{1}=35$ days, $\mu=1 / 26$ day $^{-1}$. The quality of fit: standard error of regression $s=3.6 \times 10^{-3}, R^{2}=0.998$.

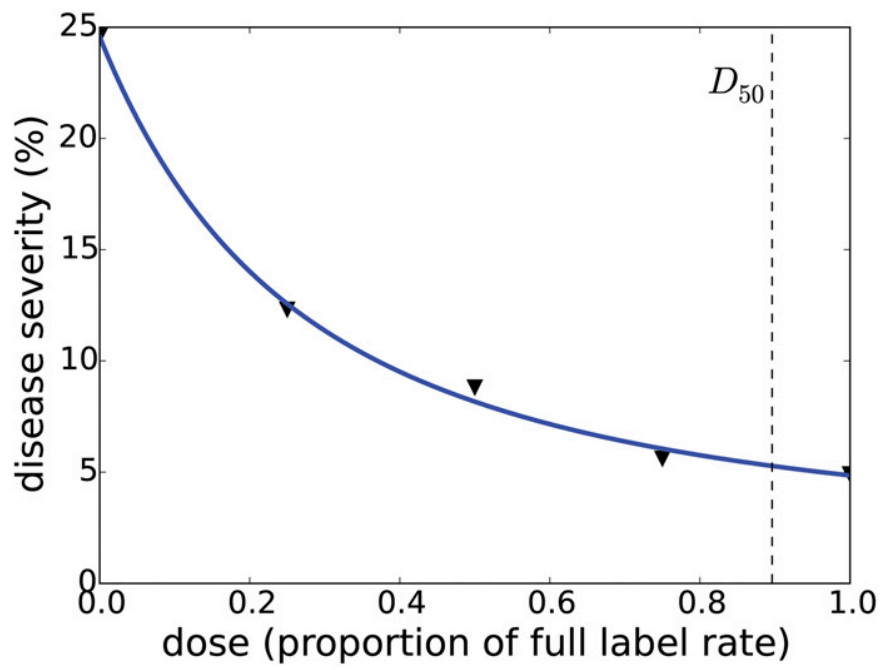

Fig. 11. Severity of Zymoseptoria tritici as a function of the epoxiconazole (Opus) dose. Points are the results of field experiments reported in (Paveley et al. 1998). The curve represents the best fit to the data points assuming exponential increase of disease severity (according to equation 55) and that the transmission rate $\beta$ decreases with the dose according to the Hill function. The best-fit parameter values: $D_{50}=0.9 \pm 0.1, \varepsilon_{m}=0.62 \pm 0.05, y_{0}=(6.12 \pm$ $0.06) \times 10^{-3}$. The value of the basic reproductive number without fungicide was taken to be $R_{0}=4.0, t_{1}=32$ days, $\mu=1 / 26$ day $^{-1}$. The quality of fit: standard error of regression $s=5.8 \times 10^{-3}, R^{2}=0.997$. 


\section{APPENDIX D. EFFECT OF PATHOGEN IMMIGRATION}

Taking into account immigration $\left(\delta_{s}>0\right.$ in equation 2$)$ leads to a more complicated expression for the rate of emergence of resistance:

$$
\begin{aligned}
\rho(D) & =\frac{1}{2 C} \frac{\varepsilon_{s}(D)}{1-\varepsilon_{s}(D)}\left[\left(R_{0}\left[1-\varepsilon_{s}(D)\right]-1\right)+\frac{\sqrt{A}}{r_{H} \mu}\right] \\
& +\delta_{s} \frac{1}{2 C} \frac{R_{0}(\mu+2 r)}{r_{H} \mu K} \varepsilon_{s}(D)
\end{aligned}
$$

where

$$
\begin{aligned}
A & =4 \beta \delta_{s} r_{H}\left(\mu+r_{H}\right)\left(1-\varepsilon_{s}(D)\right) \\
& +\left(\mu R_{0}\left(1-\varepsilon_{s}(D)\right)\left(\delta_{s}+r_{H} K\right) / \mathrm{K}-\mathrm{r}_{\mathrm{H}} \mu\right)^{2}
\end{aligned}
$$

Immigration can have a different effect on the rate of resistance emergence, depending on the parameter range (as explained in the subsection "Pathogen immigration" of the "Results" section in the main text).

A more subtle qualitative effect of immigration manifests at low fungicide doses. Without immigration and fitness costs of resistance, the resistant and sensitive pathogen strains have the same fitness at zero fungicide dose, $D=0$. As soon as the fungicide is applied, i.e., $D>0$, resistant pathogen strain acquires a fitness advantage over the sensitive strain. Immigration shifts the balance in the competition between the resistant and sensitive pathogen strains to higher fungicide doses: a range of small doses, $0<D<$ $D_{c m}$, appears, over which the sensitive strain is fitter than the resistant strain. In this respect, this effect of immigration is similar to the effect of the fitness cost of a resistance mutation. We find the threshold fungicide dose, $D_{c m}$, by solving the equation $\rho\left(D=D_{c m}\right)=$ 0 , where $\rho(D)$ is given by equation 56 :

$$
D_{c m}=\frac{\beta D_{50} \delta_{s}\left(\mu+r_{H}\right)}{\beta r_{H}\left(\varepsilon_{m} K \mu-\delta_{s}\right)-\beta \delta_{s} \mu\left(1-\varepsilon_{m}\right)-\varepsilon_{m} \mu^{2} r_{H}}
$$

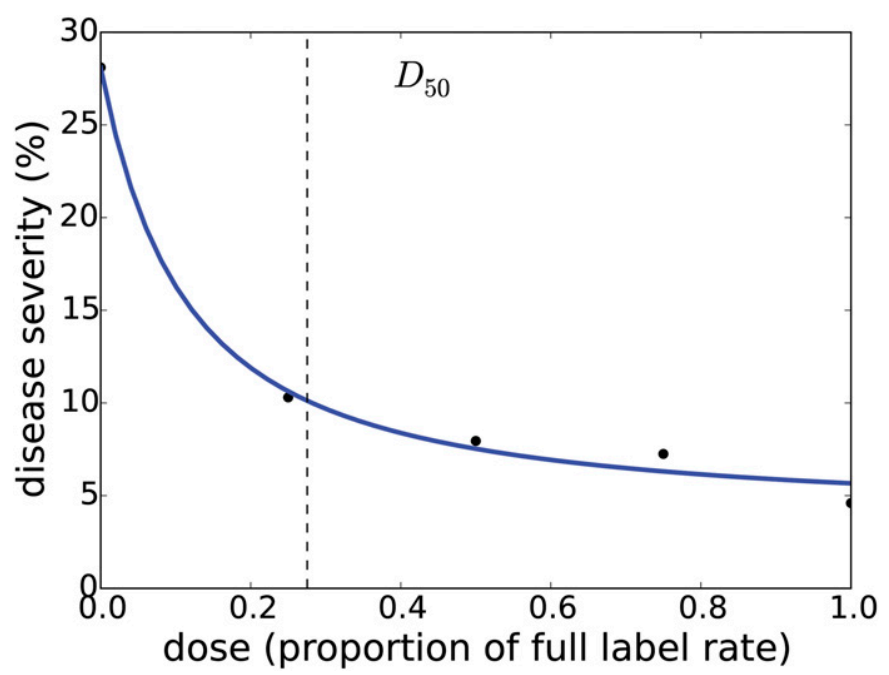

Fig. 12. Severity of Zymoseptoria tritici as a function of the pyraclostrobin (Vivid) dose. Points are the results of field experiments reported in (Lockley and Clark 2005). The curve represents the best fit to the data points assuming exponential increase of disease severity (according to equation 55) and that the transmission rate $\beta$ decreases with the dose according to the Hill function. The best-fit parameter values: $D_{50}=0.28 \pm 0.06, \varepsilon_{m}=0.44 \pm 0.03, y_{0}=(8.3 \pm$ $0.1) \times 10^{-3}$. The value of the basic reproductive number without fungicide was taken to be $R_{0}=4.0, t_{1}=30$ days, $\mu=1 / 26$ day $^{-1}$. The quality of fit: standard error of regression $s=0.01, R^{2}=0.993$.
Note, that $D_{c m}$ is proportional to $\delta_{s}$ at small values of $\delta_{s}$, hence $D_{c m}=0$ at $\delta_{s}=0$.

\section{APPENDIX E. MODEL OF PARTIAL RESISTANCE}

In the case of partial resistance, the fungicide still has an effect on the resistant mutants, but the effect is smaller than on the sensitive pathogen strain. Hence, the transmission rate of the resistant strain depends on the drug dose:

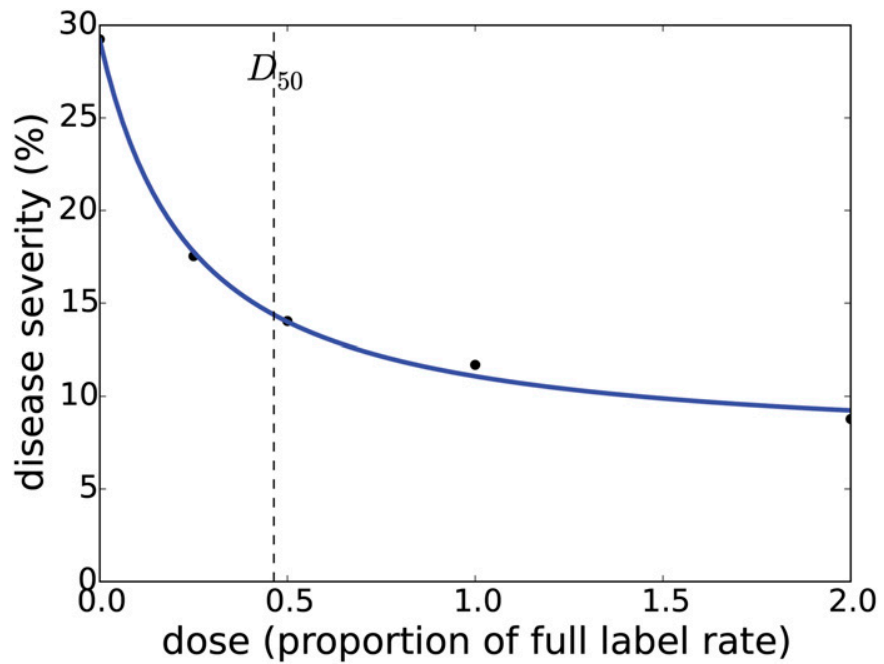

Fig. 13. Severity of Zymoseptoria tritici as a function of the azoxystrobin (Amistar) dose. Points are the results of field experiments reported in (Lockley and Clark 2005). The curve represents the best fit to the data points assuming exponential increase of disease severity (according to equation 55) and that the transmission rate $\beta$ decreases with the dose according to the Hill function. The best-fit parameter values: $D_{50}=0.46 \pm 0.04, \varepsilon_{m}=0.29 \pm 0.01, y_{0}=(7.71 \pm$ $0.06) \times 10^{-3}$. The value of the basic reproductive number without fungicide was taken to be $R_{0}=4, t_{1}=31.5$ days, $\mu=1 / 26$ day $^{-1}$. The quality of fit: standard error of regression $s=5.7 \times 10^{-3}, R^{2}=0.997$.

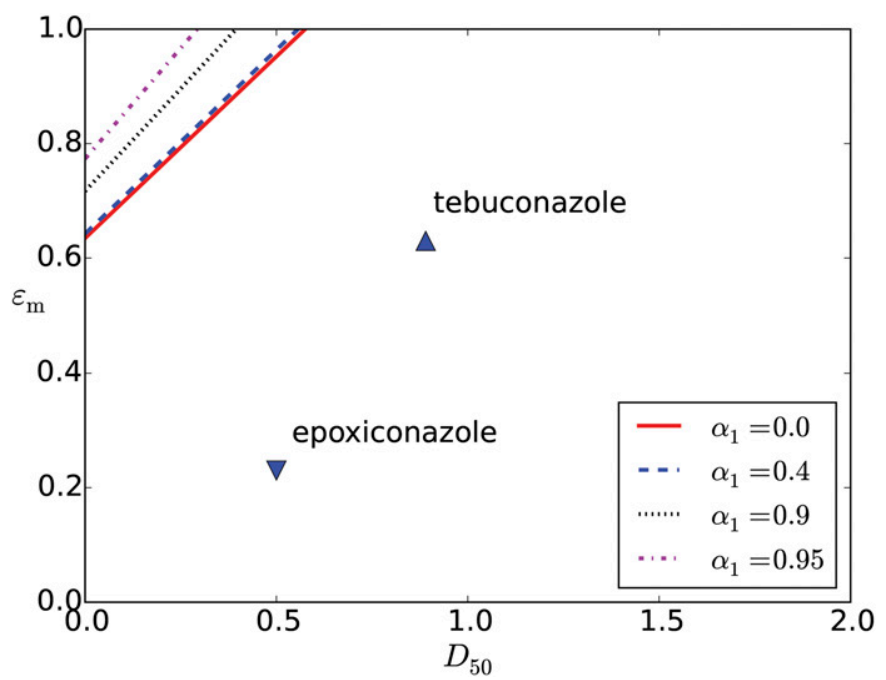

Fig. 14. The effect of type 1 partial resistance $\left(0<\alpha_{1}<1, \alpha_{2}=1\right)$ on the qualitative behavior of $T_{\text {emerg }}(D)$. Different curves indicate threshold values of maximum fungicide efficacy, $\varepsilon_{m}$, as functions of the dose-response parameter $D_{50}$ at different values of fungicide sensitivity $\alpha_{1}$ (see legend). The parameter region, where the dependence $T_{\text {emerg }}(D)$ exhibits a minimum at a fungicide dose $D_{\min }$ smaller than the maximum recommended dose $D_{m}=1$, lies to the left and above the curves. Filled triangles indicate the estimates for the doseresponse parameters $\varepsilon_{m}$ and $D_{50}$ for tebuconazole and epoxiconazole. The curves were plotted according to equation 44 with the parameter values $R_{0}=4$, $\theta=0.15$. 


$$
\beta_{r}=\beta\left[1-\varepsilon_{r}(D)\right]
$$

where

$$
\varepsilon_{r}(D)=\alpha_{1} \varepsilon_{m} \frac{D}{D+D_{50} / \alpha_{2}}
$$

is the fungicide efficacy for the resistant strain. Resistance may affect the dose-response dependence in two different ways: (i) by decreasing $\varepsilon_{m}$, when fungicide sensitivity $0<\alpha_{1}<1$ and $\alpha_{2}=1$ or (ii) by increasing $D_{50}$, when fungicide sensitivity $0<\alpha_{2}<1$ and $\alpha_{1}=1$. In a more general case, these two types of partial resistance manifest together, i.e., $0<\alpha_{1}, \alpha_{2}<1$.

We obtain the expression for the probability of mutant survival by substituting equation 3 , equation 5 (at $\theta=0$ ), equation 26 and equation 31 in equation 30 for the case when $R_{0 s}>1$ :

$$
P_{\text {surv }}=\frac{\varepsilon_{s}(D)-\varepsilon_{r}(D)}{1-\varepsilon_{r}(D)}
$$

The expression for the rate of mutant appearance in the case of partial resistant is determined by substituting equation 60 in equation 33:

$$
r_{a p p}=\frac{1}{C} \frac{\left[1-\varepsilon_{r}(D)\right]\left(R_{0}\left[1-\varepsilon_{s}(D)\right]-1\right)}{\left[1-\varepsilon_{s}(D)\right]}
$$

where $C$ is given by equation 35 . Then, the probability of resistance emergence per time unit reads

$$
\rho(D)=r_{\text {app }} P_{\text {surv }}=\frac{1}{C} \frac{\left(R_{0}\left[1-\varepsilon_{s}(D)\right]-1\right)\left[\varepsilon_{s}(D)-\varepsilon_{r}(D)\right]}{\left[1-\varepsilon_{s}(D)\right]}
$$

Consequently, the mean waiting time has the expression

$$
T_{\text {emerg }}=1 / \rho=C \frac{\left[1-\varepsilon_{S}(D)\right]}{\left(R_{0}\left[1-\varepsilon_{s}(D)\right]-1\right)\left[\varepsilon_{s}(D)-\varepsilon_{r}(D)\right]}
$$

Partial resistance type 1 (PR1). First, consider partial resistance of type 1 (PR1), when resistance only affects the asymptote, $\varepsilon_{m}\left(0<\alpha_{1}<1, \alpha_{2}=1\right)$. We obtain the expression for the time until

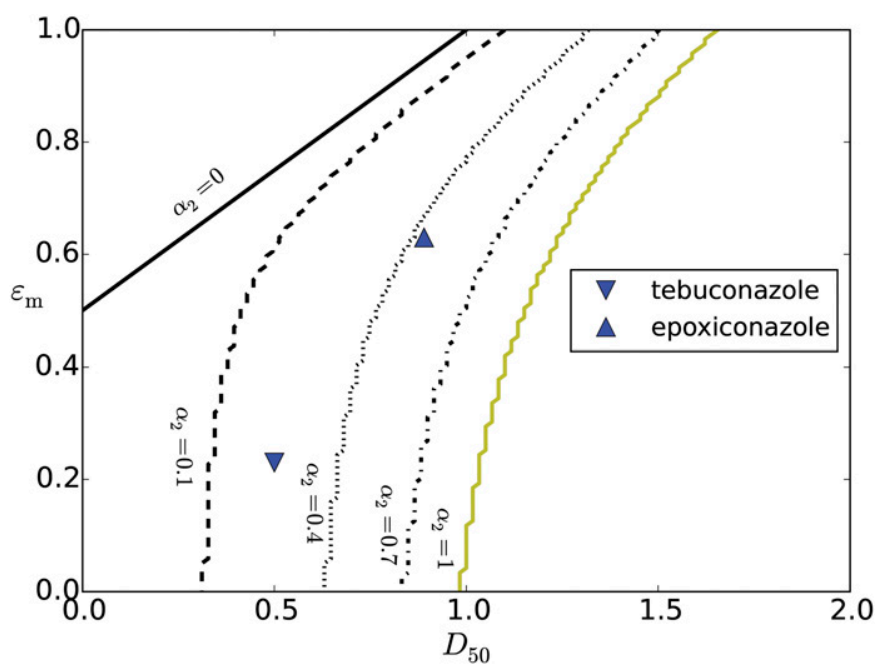

Fig. 15. The effect of type 2 partial resistance $\left(\alpha_{1}=1,0<\alpha_{2}<1\right)$ on the qualitative behavior of $T_{\text {emerg }}(D)$. The curves show the contours $D_{\text {min }}=1$ versus $\varepsilon_{m}$ and $D_{50} ; D_{\min }<1$ in the region to the left from the curves. The solid black curve corresponds to full resistance, i.e., $\alpha_{2}=0$. Other curves correspond to different values of $\alpha_{2}, 0<\alpha_{2}<1$ (see graph). The curves were obtained numerically using $R_{0}=4, \theta=0, \delta_{s}=0$ (Appendix E). Filled triangles indicate the estimates for the dose-response parameters $\varepsilon_{m}$ and $D_{50}$ for tebuconazole and epoxiconazole. emergence by substituting $\alpha_{2}=1$ in equation 64. With this expression, we performed the analysis similar to the one done in Appendix B. In particular, we solved the equation $d T_{\text {emerg }}(D) / d D=$ 0 with respect to $D$. We found that in the absence of the fitness cost (at $\theta=0$ ) the fungicide dose at which the minimum emergence time is reached is given by the same expression as in the case of absolute resistance, equation 11 . The dose-response curve of resistant pathogens is merely shifted down along the vertical axis with respect to the dose-response curve of sensitive pathogens, i.e., $\varepsilon_{r}(D)=\alpha_{1} \varepsilon_{s}(D)$. This may affect the dependencies $r_{a p p}(D)$ and $P_{\text {surv }}(D)$ differently for different doses. However, the final expression for the rate of resistance emergence, $\rho(D)$ (equation 7), is proportional to $\varepsilon_{s}(D)-\varepsilon_{r}(D)=\left(1-\alpha_{1}\right) \varepsilon_{s}(D)$. As a result, PR1 affects the rate of emergence, $\rho$, in the same way for all fungicide doses. Importantly, PR1 does not change the position of the minimum, $D_{\text {min }}$, in the time until emergence, $T_{\text {emerg }}(D)$, versus the fungicide dose, $D$. Therefore, the range over which $D_{\min }<1$ remains the same as in the case of the full resistance (Fig. 4). In contrast, when the fitness cost is present $(\theta>0)$, PR 1 shifts the maximum in such a way that the parameter range over which $D_{\text {min }}<1$ is reduced (Fig. 14).

Partial resistance type 2 (PR2). Next, we investigate the case of partial resistance of type 2 (PR2), when it only affects the shape parameter $D_{50}\left(\alpha_{1}=1,0<\alpha_{2}<1\right)$. Here, in contrast to PR1, the maximum in the probability of mutant survival, $P_{\text {surv }}(D)$ appears in the whole range of values of $\varepsilon_{m}, D_{50}$, and $R_{0}$, i.e., $0<\varepsilon_{m}<1, D_{50}>0$, and $R_{0}>1$. The analysis of the expression for $P_{\text {surv }}(D)$ in equation 61 reveals that the maximum is reached at the dose $D=D_{\text {max }}^{*}$, where

$$
D_{\max }^{*}=\frac{D_{50}}{\sqrt{\alpha_{2}\left(1-\varepsilon_{m}\right)}}
$$

Note that the expression for $D_{\text {max }}^{*}$ in equation 65 is proportional to $D_{50}$, depends on $\varepsilon_{m}$ and $\alpha_{2}$, but is independent of $R_{0}$. The maximum in $P_{\text {surv }}(D)$ leads to a minimum in $T_{\text {emerg }}(D)$ in the whole range of parameter values, because $T_{\text {emerg }}(D) \propto 1 / P_{\text {surv }}(D)$.

For practical applications, the position of the minimum on the dose axis, $D_{\text {min }}$, is important. The range of doses over which emergence takes longer at larger doses can only be used in practice, if it lies below the maximum recommended dose $D_{\text {rec }}=1$. Therefore, we are interested in finding parameter ranges over which the dose $D_{\min }$ is smaller than $D_{\text {rec }}=1$. Specifically, we determine the ranges of parameters $R_{0}, \varepsilon_{m}, D_{50}$, and $\alpha_{2}$ over which this requirement is fulfilled. Here, it is not possible to obtain analytical expressions for the boundaries of these parameter ranges. Hence, we performed numerical analysis using the following algorithm:

1. Solve the equation $\frac{d \rho(D)}{d D}=0$ with respect to $D$, to find $D_{\text {min }}$ for each set of parameter values of interest.

2. Calculate $\frac{d^{2} \rho}{d D^{2}}\left(D=D_{\min }\right)$. If the value is negative, then $D_{\min }$ corresponds to the maximum in $\rho(D)$ (minimum in $T_{\text {emerg }}(D)$ ).

3. Solve the equation $D_{\text {min }}=D_{\text {rec }}$, in order to determine boundaries of parameter regions, where $D_{\min }<D_{\text {rec }}$.

4. Plot these boundaries as functions of parameters $\varepsilon_{m}, D_{50}, \alpha_{2}$, and $R_{0}$.

Using this approach, we plotted the borders of this range (contours of $D_{\min }=1$ ) first versus $\varepsilon_{m}$ and $R_{0}$ (Fig. 5), and then also versus $\varepsilon_{m}$ and $D_{50}$ (Fig. 15) at different values of $\alpha_{2}$. The regions where $D_{\min }<1$ are located to the left from and above the curves in Figures 5 and 15 .

In Figures 5 and 15, black curves of different styles correspond to different values of the fungicide sensitivity $\alpha_{2}$. At full resistance $\left(\alpha_{2}=0\right.$, solid black curve), the condition $D_{\min }<1$ is fulfulled in a narrow parameter range corresponding to large values of $\varepsilon_{m}$ and small values of $D_{50}$. For partial resistance of type 2 , at $\alpha_{2}>0$, the parameter range where $D_{\min }<1$ extends to include small values of $\varepsilon_{m}$. The curve corresponding to the threshold $D_{\min }=1$ shifts toward larger values of $D_{50}$ as the fungicide sensitivity, $\alpha_{2}$, increases. 
A fungicide-resistant strain is viable only when the fitness cost is small enough, such that there exists a range of fungicide doses over which $P_{\text {surv }}>0$. In this range of fitness cost values, its presence does not affect the position of the maximum in $P_{\text {surv }}(D)$ and influences only marginally the position of the maximum in $r_{a p p}(D)$. Therefore, the presence of a fitness cost does not change our conclusions about the effect of PR2 in any significant way.

The effect of combined partial resistance type 1 and type 2. Next, we investigate the effect of the combined partial resistance of type 1 and type 2 on the dose dependence of the probability of survival and the time until resistance emergence. When the two types of partial resistance are present together, the probability of survival achieves a maximum at the dose

$$
D_{\max 12}^{*}=\frac{D_{50}}{\alpha_{2}} \frac{\alpha_{2}\left(1-\alpha_{1}\right)+\sqrt{\alpha_{1} \alpha_{2}\left(1-\alpha_{2}\right)\left(1-\varepsilon_{m}-\alpha_{2}\left(1-\alpha_{1} \varepsilon_{m}\right)\right)}}{\alpha_{1}\left(1-\varepsilon_{m}\left[1-\alpha_{2}\right]\right)-\alpha_{2}}
$$

where we assumed no fitness costs, i.e., $\theta=0 . D^{*}{ }_{\max 12}$ in equation 66 is positive for large enough values of the fungicide sensitivity $\alpha_{1}$, i.e., the condition $\alpha_{1 c}<\alpha_{1}<1$ is fulfilled, where the threshold fungicide sensitivity has the expression

$$
\alpha_{1 c}=\frac{\alpha_{2}}{1-\varepsilon_{m}\left(1-\alpha_{2}\right)}
$$

The analysis of equation 67 indicates that the range of $\alpha_{1}$ values over which the maximum in $P_{\text {surv }}(D)$ exists for positive fungicide doses decreases with increasing $\alpha_{2}$ and $\varepsilon_{m}$.

As we are interested in the parameter regions over which $D^{*}{ }_{\max 12}<1$, we calculated the border value of the fungicide sensitivity, $\alpha_{2}$ at which $D^{*}{ }_{\max 12}=1$ :

$$
\alpha_{1 b}=\frac{\left(\alpha_{2}+D_{50}\right)^{2}}{\alpha_{2}\left(\left(1+D_{50}\right)^{2}-\varepsilon_{m}\left(1-\alpha_{2}\right)\right)}
$$

Figure 3 (lowest panels) illustrates the scenario, when the two types of resistance (PR1 and PR2) are present simultaneously. In this case, the fungicide effect on the resistant strain, $\varepsilon_{r}(D)$ grows slower with the dose (PR2) and reaches a lower asymptote at high

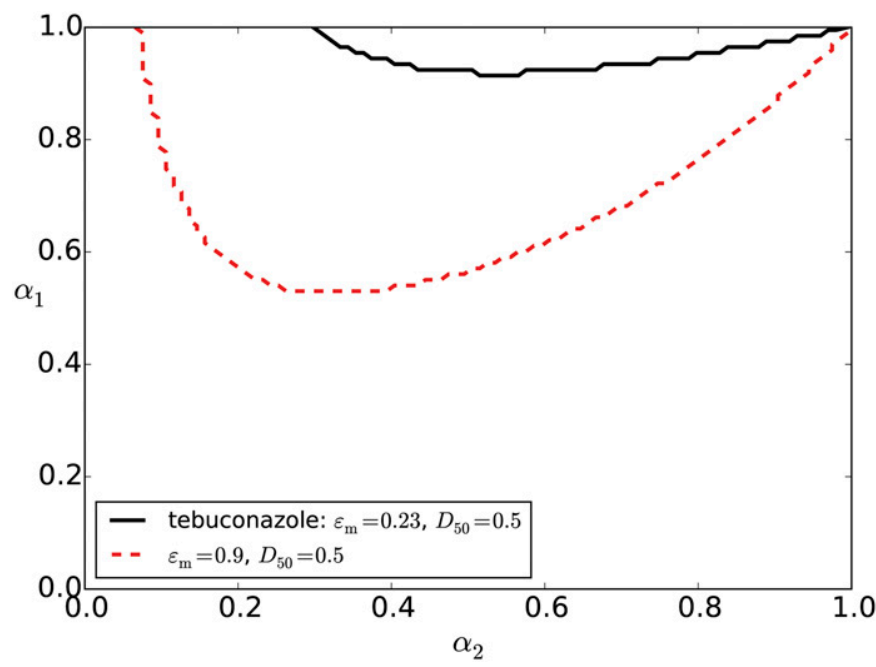

Fig. 16. Combined effect of partial resistance type 1 and type $2\left(0<\alpha_{1}<1<\right.$ $\alpha_{2}<1$ ) on the qualitative behavior of $T_{\text {emerg }}(D)$. Curves indicate contours $D_{\text {min }}=1$ versus fungicide sensitivities $\alpha_{1}$ and $\alpha_{2}$ at $\varepsilon_{m}=0.23$ (estimate for tebuconazole, solid line) and $\varepsilon_{m}=0.9$ (dashed line). $T_{\text {emerg }}(D)$ exhibits a minimum at a fungicide dose $D_{\min }<1$ in the parameter region above the curves. Parameter values: $R_{0}=4, D_{50}=0.5$. doses (PR1). In this case, there may still be a maximum in the probability of survival versus dose, $P_{\text {surv }}(D)$, as in the case of PR2, but here the maximum is shifted to higher doses and becomes less pronounced due to the difference in the asymptotes (Fig. 3, lowest right panel).

Therefore, when the effect of PR1 is added on top of PR2, we expect the maximum in $P_{\text {surv }}(D)$ to shift to higher doses. Consequently, the minimum in $T_{\text {emerg }}(D)$ will shift to higher doses and will eventually exceed the maximum recommended dose, when the difference between the asymptotes becomes large enough (i.e., at small enough $\alpha_{1}$ values).

We quantified this effect using numerical analysis and illustrated the outcomes in Figure 16. This figure shows the ranges of fungicide sensitivities $\alpha_{1}$ and $\alpha_{2}$ over which the minimum in $T_{\text {emerg }}$ is achieved below the label recommended dose (at $D_{\min }<1$ ). The curves indicate the contours $D_{\min }=1$, the range where $D_{\min }<1$ lies above the curves, and the range where $D_{\min }>1$ lies below the curves. As expected, we see from Figure 16 that at small values of $\alpha_{1}$ the minimum in $T_{\text {emerg }}(D)$ exceeds the label recommended dose $\left(D_{\min }>1\right)$. Figure 16 shows more quantitative details of this effect: $D_{\min }<1$ is fulfilled over a range of intermediate to large $\alpha_{1}$ values and intermediate $\alpha_{2}$ values. The size of this region increases with increasing maximum fungicide efficacy $\varepsilon_{m}$ (Fig. 16, compare solid and dashed curves).

\section{ACKNOWLEDGMENTS}

A. Mikaberidze and S. Bonhoeffer gratefully acknowledge financial support by the ERC advanced grant PBDR 268540 "The population biology of drug resistance: Key principles for a more sustainable use of drugs". A. Mikaberidze is grateful to the Swiss National Science Foundation for financial support through the Ambizione Junior Group Leader grant PZOOP3_161453. Rothamsted Research receives support of the Biotechnology and Biological Research Council (BBSRC) of the U.K. A. Mikaberidze and S. Bonhoeffer are grateful to H. Alexander, B. McDonald, and members of the Theoretical Biology group at the ETH Zurich for fruitful discussions and brainstorming.

\section{LITERATURE CITED}

Anderson, R. M., and May, R. M. 1986. The invasion, persistence and spread of infectious diseases within animal and plant communities. Philos. Trans. R. Soc. Lond. B Biol. Sci. 314:533-570.

Brent, K. J., and Hollomon, D. W. 2007. Fungicide Resistance in Plant Management: How can it be managed? FRAC Monograph No. 1. Fungicide Resistance Action Committee. http://www.frac.info/

Caswell, H. 2001. Matrix Population Models. Sinauer Associates, Sunderland, MA

Cools, H. J., and Fraaije, B. 2013. Update on mechanisms of azole resistance in Mycosphaerella graminicola and implications for future control. Pest Manag. Sci. 69:150-155.

Cunniffe, N. J., and Gilligan, C. a. 2010. Invasion, persistence and control in epidemic models for plant pathogens: The effect of host demography. J. R. Soc. Interface 7:439-451.

Day, T., and Read, A. F. 2016. Does high-dose antimicrobial chemotherapy prevent the evolution of resistance? PLOS Comput. Biol. 12:e1004689.

Dean, R., Van Kan, J. A. L., Pretorius, Z. A., Hammond-kosack, K. I. M. E., Pietro, A. D. I., Spanu, P. D., Rudd, J. J., Dickman, M., Kahmann, R., Ellis, J., and Foster, G. D. 2012. The top 10 fungal pathogens in molecular plant pathology. Mol. Plant Pathol. 13:414-430.

Drlica, K., and Zhao, X. 2007. Mutant selection window hypothesis updated. Clin. Infect. Dis. 44:681-688.

Eyal, Z. 1971. The kinetics of pycnospore liberation in Septoria tritici. Can. J. Bot. 49:1095-1099.

Grimmer, M. K., van den Bosch, F., Powers, S. J., and Paveley, N. D. 2014. Fungicide resistance risk assessment based on traits associated with the rate of pathogen evolution. Pest Manag. Sci. 71:207-215.

Hall, R. J., Gubbins, S., and Gilligan, C. A. 2007. Evaluating the performance of chemical control in the presence of resistant pathogens. Bull. Math. Biol. 69:525-537.

Hallatschek, O., and Nelson, D. R. 2009. Life at the front of an expanding population. Evolution (N.Y.) 64:193-206.

Hobbelen, P. H. F., Paveley, N. D., and Bosch, F. V. D. 2014. The emergence of resistance to fungicides. PLoS ONE 9:e91910. 
Hobbelen, P. H. F., Paveley, N. D., and van den Bosch, F. 2011. Delaying selection for fungicide insensitivity by mixing fungicides at a low and high risk of resistance development: A modeling analysis. Phytopathology 101: 1224-1233.

Huijben, S., Bell, A. S., Sim, D. G., Tomasello, D., Mideo, N., Day, T., and Read, A. F. 2013. Aggressive chemotherapy and the selection of drug resistant pathogens. PLoS Pathog. 9:e1003578.

Jorgensen, L. N., Hovmoller, M. S., Hansen, J., Lassen, P., Clark, B., Bayles, R., Rodemann, B., Flath, K., Jahn, M., Goral, T., Jerzy Czembor, J., Cheyron, P., Maumene, C., De Pope, C., Ban, R., Nielsen, G. C., and Berg, G. 2014. IPM strategies and their dilemmas including an introduction to www.eurowheat.org. J. Integr. Agric. 13:265-281.

Kouyos, R. D., Metcalf, C. J. E., Birger, R., Klein, E. Y., Abel zur Wiesch, P., Ankomah, P., Arinaminpathy, N., Bogich, T. L., Bonhoeffer, S., Brower, C., Chi-Johnston, G., Cohen, T., Day, T., Greenhouse, B., Huijben, S., Metlay, J., Mideo, N., Pollitt, L. C., Read, A. F., Smith, D. L., Standley, C., Wale, N., and Grenfell, B. 2014. The path of least resistance: Aggressive or moderate treatment? Proc. R. Soc. B Biol. Sci. 281:1-8.

Linde, C. C., Zhan, J., and McDonald, B. A. 2002. Population structure of Mycosphaerella graminicola: From lesions to continents. Phytopathology 92:946-955.

Lindsey, H. A., Gallie, J., Taylor, S., and Kerr, B. 2013. Evolutionary rescue from extinction is contingent on a lower rate of environmental change. Nature 494:463-467.

Lion, S., and Gandon, S. 2015. Evolution of spatially structured host-parasite interactions. J. Evol. Biol. 28:10-28.

Lockley, D., and Clark, W. S. 2005. Fungicide dose-response trials in wheat: The basis for choosing "appropriate dose". Home-Grown Cereal. Authority, UK, Proj. Rep. 373.

Madden, L. V., Hughes, G., and van den Bosch, F. 2006a. Temporal Analysis II: The Components of Disease. Chapter 5, pages 117-143 in: Study of Plant Disease Epidemics. American Phytopathological Society, St. Paul, MN.

Madden, L. V., Hughes, G., and van den Bosch, F. 2006b. Temporal Analysis III: Advanced Topics. Chapter 6 in: Study of Plant Disease Epidemics. American Phytopathological Society, St. Paul, MN.

Miguez, M., Reeve, C., Wood, P. M., and Hollomon, D. W. 2004. Alternative oxidase reduces the sensitivity of Mycosphaerella graminicola to QOI fungicides. Pest Manag. Sci. 60:3-7.

Mikaberidze, A., McDonald, B., and Bonhoeffer, S. 2015. Developing smarter host mixtures to control plant disease. Plant Pathol. 64:996-1004.
Mikaberidze, A., McDonald, B. A., and Bonhoeffer, S. 2014. Can high risk fungicides be used in mixtures without selecting for fungicide resistance? Phytopathology 104:324-331.

Mikaberidze, A., Mundt, C. C., and Bonhoeffer, S. 2016. Invasiveness of plant pathogens depends on the spatial scale of host distribution. Ecol. Appl. 26: 1238-1248.

Milgroom, M. G. 1990. A stochastic model for the initial occurrence and development of fungicide resistance in plant pathogen populations. Phytopathology 80:410-416.

Paveley, N., Hims, M., and Stevens, D. 1998. Appropriate fungicide doses for winter wheat and matching crop management to growth and yield potential. Home-Grown Cereal. Authority, UK, Proj. Rep. 166.

Plantegenest, M., Le May, C., and Fabre, F. 2007. Landscape epidemiology of plant diseases. J. R. Soc. Interface 4:963-972.

Read, A. F., Day, T., and Huijben, S. 2011. The evolution of drug resistance and the curious orthodoxy of aggressive chemotherapy. Proc. Natl. Acad. Sci. 108:10871-10877.

Robert, C., Fournier, C., Andrieu, B., and Ney, B. 2008. Coupling a 3D virtual wheat (Triticum aestivum) plant model with a Septoria tritici epidemic model (Septo3D): A new approach to investigate plant-pathogen interactions linked to canopy architecture. Funct. Plant Biol. 35:997-1013.

Roberts, J. A., Kruger, P., Paterson, D. L., and Lipman, J. 2008. Antibiotic resistance-what's dosing got to do with it? Crit. Care Med. 36:2433-2440.

Roques, L., Garnier, J., Hamel, F., and Klein, E. K. 2012. Allee effect promotes diversity in traveling waves of colonization. Proc. Natl. Acad. Sci. 109:8828-8833.

Shaw, M. 1994. Modeling stochastic processes in plant pathology. Annu. Rev. Phytopathol. 32:523-544.

Shaw, M. W. 1989. A model of the evolution of polygenically controlled fungicide resistance. Plant Pathol. 38:44-55.

van den Bosch, F., and Gilligan, C. A. 2008. Models of fungicide resistance dynamics. Annu. Rev. Phytopathol. 46:123-147.

van den Bosch, F., Oliver, R., van den Berg, F., and Paveley, N. 2014. Governing principles can guide fungicide-resistance management tactics. Annu. Rev. Phytopathol. 52:175-195.

van den Bosch, F., Paveley, N., Shaw, M., Hobbelen, P., and Oliver, R. 2011. The dose rate debate: Does the risk of fungicide resistance increase or decrease with dose? Plant Pathol. 60:597-606.

zur Wiesch, P. A., Kouyos, R., Engelstädter, J., Regoes, R. R., and Bonhoeffer, S. 2011. Population biological principles of drug-resistance evolution in infectious diseases. Lancet Infect. Dis. 11:236-247. 